OPEN ACCESS

Edited by:

Gordon A. Francis,

Simon Fraser University, Canada

Reviewed by:

Rhys David Evans,

University of Oxford, United Kingdom Mireille Ouimet,

University of Ottawa, Canada

*Correspondence:

Kimberley D. Bruce

kimberley.bruce@

cuanschutz.edu

Specialty section:

This article was submitted to Lipid and Fatty Acid Research, a section of the journal Frontiers in Physiology

Received: 13 January 2020 Accepted: 02 April 2020 Published: 28 April 2020

Citation: Loving BA and Bruce KD (2020) Lipid and Lipoprotein Metabolism in Microglia. Front. Physiol. 11:393. doi: 10.3389/fphys.2020.00393

\section{Lipid and Lipoprotein Metabolism in Microglia}

\author{
Bailey A. Loving ${ }^{1}$ and Kimberley D. Bruce ${ }^{2 *}$ \\ ${ }^{1}$ School of Medicine, University of Colorado, Anschutz Medical Campus, Aurora, CO, United States, ${ }^{2}$ Division \\ of Endocrinology, Metabolism and Diabetes, University of Colorado, Anschutz Medical Campus, Aurora, CO, United States
}

Microglia, once viewed as static bystanders with limited homeostatic functions, are now considered key players in the development of neuroinflammatory and neurodegenerative diseases. Microglial activation is a salient feature of neuroinflammation involving a dynamic process that generates multitudinous microglial phenotypes that can respond to a variety of situational cues in the central nervous system. Recently, a flurry of single cell RNA-sequencing studies have defined microglial phenotypes in unprecedented detail, and have highlighted robust changes in the expression of genes involved in lipid and lipoprotein metabolism. Increased expression of genes such as Apolipoprotein E (ApoE), Triggering Receptor Expressed on Myeloid Cells 2 (TREM2) and Lipoprotein Lipase (LPL) in microglia during development, damage, and disease, suggest that increased lipid metabolism is needed to fuel protective cellular functions such as phagocytosis. This review describes our current understanding of lipid and lipoprotein metabolism in microglia, and highlights microglial lipid metabolism as a modifiable target for the treatment of neurodegenerative diseases such as Alzheimer's disease and multiple sclerosis.

Keywords: lipid and lipoprotein metabolism, microglia, neurodegenerating diseases, Alzheimer's disease, lipid, lipoprotein, multiple scleorsis (MS), APOE

\section{INTRODUCTION}

Microglia are functionally distinct brain-resident macrophages that are seeded developmentally and maintained by self-proliferation (Ajami et al., 2007; Alliot et al., 1991; Askew et al., 2017). Once considered static bystanders with limited homeostatic functions, it is now becoming increasingly clear that microglia interact with all CNS components and have a marked impact on brain health and disease ( $\mathrm{Li}$ and Barres, 2018). Microglia are highly plastic and respond to a variety of environmental cues by switching to appropriate activation states. While some activation states are adaptive and contribute to homeostatic functions, others are maladaptive and associated with neuroinflammation. Microglial activation and dysfunction are salient features of neuroinflammatory and neurodegenerative diseases (NDs), such as Alzheimer's disease (AD), Parkinson's disease (PD), and multiple sclerosis (MS) (Colonna and Butovsky, 2017). Thus, it has been increasingly recognized that understanding the heterogeneity of microglial activation in the context of disease may facilitate the design of therapeutics that dampen the detrimental effects of microglial activation, while augmenting the beneficial effects of "alternatively" activated microglia (Li and Barres, 2018). 
Recently, several comprehensive single-cell RNA-sequencing (scRNAseq) analyses of microglia ex vivo have defined the transcriptomic identities of microglia with temporal (Grabert et al., 2016; Hammond et al., 2019; Li et al., 2019), regional (Grabert et al., 2016; Hammond et al., 2019; Li et al., 2019), and disease state specificity (Hammond et al., 2019; Keren-Shaul et al., 2017; Mathys et al., 2017). Various clusters of microglia with similar gene expression profiles have been mapped to specific regions and developmental or disease stages. A striking

Abbreviations: 11ß-HSD1, 11 -hydroxysteroid dehydrogenase type 1; 15d$\mathrm{PGJ}_{2}$, 15-deoxy- $\Delta^{12-14}-\mathrm{PGJ}_{2} ;$ 18-HEPE, 18-hydroxyeicosapentaenoic acid; 24-OHC, 24(S)-hydroxycholesterol; 5XFAD, 5 familial AD mutations; 7KC, 7-ketocholesterol; $\mathrm{A} \beta$, amyloid beta; AA, arachidonic acid; ABC, ATP-binding cassette transporter; ACAT, Sterol O-acyltransferase; acyl-CoA, acyl-coenzyme A; AD, Alzheimer's disease; AIBP, ApoA-1 binding protein; Akt, Protein kinase B; ALA, alpha-linolenic acid; ApoA-1, apolipoprotein A-1; ApoB-100, apolipoprotein B-100; ApoE, Apolipoprotein E; ApoJ, apolipoprotein J; APP, Amyloid precursor protein; ARG1, Arginase 1; ATGL, Adipose triglyceride lipase; ATP, adenosine triphosphate; BAM, Border-Associated Microglia; BBB, blood brain barrier; CD11b/CD18, C3 receptor; CE, cholesterol ester; CLEM, Correlative light and electron microscopy; CLU, Clusterin; CM, chylomicron; CNS, central nervous system; COX2, cyclooxygenase 2; cPLA2, cytosolic group IV of PLA2; CPT1A, Carnitine Palmitoyltransferase 1A; CSF, cerebrospinal fluid; CSF-1R, Colony stimulating factor 1 receptor; CX3CL1, chemokine fractalkine; CX3CR1, C-X3-C Motif Chemokine Receptor 1; CXCL1, chemokine (C-X-C motif) ligand 1; CYP46A1, cholesterol 24S-hydroxylase; DAG, diacylglycerol; DAM, disease associated microglia; DAP12, DNA polymerase III-Activation Protein 12; db/db, leptin deficient mouse model of type 2 diabetes; DHA, docosahexaenoic acid; EGFP, enhanced GFP; EPA, eicosatetraenoic acid; ER, endoplasmic reticulum; fA $\beta$, fibrillar $A \beta$; FA, fatty acid; FABP, FA binding protein; $\mathrm{FAD}$, familial AD; FAO, FA oxidation; FATP, FA transport protein; FFA, free FA; GFP, green fluorescent protein; GL, glycolipid; GPAT, Glycerol-3-phosphate acyltransferase; GPR109A, G-protein-coupled receptors 109A.; GWAS, Genome Wide Association Studies; HDL, high-density lipoprotein; HFD, high-fat diet; HMGB1, High Mobility Group Box 1; HMGCR, 3-hydroxy-3-methyl-glutarylcoenzyme A reductase; HSL, hormone sensitive lipase; Iba1, Ionized calcium binding adaptor molecule 1; IDL, intermediate-density-lipoproteins; IFN, interferon; IGF1, Insulin growth factor 1; IHC, immunohistochemistry; iMG, induced microglia-like cell culture; IP, intraperitoneal; LA, linoleic acid; LC, long chain; LCAT, Lecithin-cholesterol acyltransferase; LDL, low-density lipoprotein; LDLR, LDL receptor; LOX, lipoxygenase; LPA, lysophosphatidic acid; LPC, lysophosphatidylcholine; LPL, Lipoprotein Lipase; LPS, lipopolysaccharide; LRP1, LDL Receptor Related Protein 1; LXR, liver X receptor; $\mathrm{mA} \beta$, monomeric A $\beta$; MFG-E8, Milk fat globule epidermal growth factor-8; MFP2, multi-functional protein-2; Mfsd2a, Major Facilitator Superfamily Domain Containing 2A; MS, multiple sclerosis; MS4A7, Membrane-spanning 4-domains subfamily A member 7; mTOR, mammalian target of rapamycin; MUFA, monounsaturated FA; $\mathrm{n}-3$, omega-3; n-6, omega-6; ND, neurodegenerative disease; NF-kB, Nuclear Factor kappa-light-chain-enhancer of activated B cells; NPC, neural progenitor cell; NSC, neural stem cell; oA $\beta$, oligomeric A $\beta$; OL, oligodendrocyte; OPC, oligodendrocyte precursor cell; oxLDL, oxidized LDL; OXPHOS, oxidative phosphorylation; PAM, proliferative-region-associated-microglia; PARP, poly (ADP-ribose)-polymerase; PC, phosphatidylcholine; PD, Parkinson's disease; PE, phosphatidylethanolamine; PET, Positron emission tomography; PI3K, Phosphoinositide 3-kinase; PL, phospholipid; PLA2, phospholipase A2; PPAR, peroxisome proliferator activated receptor; PPP, pentose phosphate pathway; PS, phosphatidylserine; PS1, Presenilin-1; PSD95, postsynaptic density protein 95; PUFA, polyunsaturated FA; ROS, reactive oxygen species; RXR, retinoid X receptor; SAD, sporadic late onset $\mathrm{AD}$; SCARA, class A scavenger receptor gene; SCARB, class B scavenger receptor gene, SCARC, class C scavenger receptor gene, FAT, FA translocase; scRNAseq, single-cell RNA-sequencing; SFA, saturated fatty acid; siglec-11, sialic acid-binding Ig-like lectin; Slc2a5, Solute carrier family 2 member 5; SNAP25, Synaptosome Associated Protein 25; SPM, specialized proresolving mediator; Spp1, Secreted phosphoprotein 1; SR-B1, class B scavenger receptor protein 1; SREBP, sterol regulatory element binding proteins; STED, stimulated emission depletion; SZ, schizophrenia; TBI, traumatic brain injury; TG, triglyceride; TGF $\beta$, Transforming growth factor $\beta$; TLR, toll-like receptor; Tmem119, Transmembrane Protein 119; TREM2, Triggering Receptor Expressed on Myeloid Cells 2; VLDL, very-low-density-lipoprotein; VLDLR, VLDL receptor. feature of these studies is the identification of microglial clusters with common metabolic characteristics. For example, KerenShaul et al. (2017) used the 5XFAD murine model of AD to define Disease Associated Microglia (DAMs) that express a distinct set of genes associated with lipid and lipoprotein metabolism (e.g., Apolipoprotein E [ApoE], Lipoprotein Lipase [LPL], and Triggering Receptor Expressed On Myeloid Cells 2 [TREM2]) (Keren-Shaul et al., 2017). This transcriptional signature represents a preference for lipids as fuel substrates that fulfill the greater bioenergetics needs of activated microglia (Keren-Shaul et al., 2017). Interestingly, these DAMs bear a striking resemblance to microglial clusters observed during early postnatal life (P4/P5) (Hammond et al., 2019), and in proliferative-region-associated-microglia (PAMs), which reside in regions of the early postnatal brain with active gliogenesis and neurogenesis (Li et al., 2019). A similar signature is also observed in microglia of the demyelinating brain (Hammond et al., 2019), and in the later stages of a CK-p25 murine model of DNA damage and neurodegeneration (Mathys et al., 2017).

Even though these studies have varied in their experimental approach, they have repeatedly implicated consistent changes in microglial metabolism during microglial activation. These studies have reignited our interest in "immunometabolism," particularly in the context of neurodegenerative disease (ND). Here, we review our current understanding of lipid and lipoprotein metabolism in microglia to identify potentially targetable metabolic processes, which may ameliorate detrimental microglial responses and help develop novel therapeutic interventions for $\mathrm{ND}$ and beyond.

\section{IMPORTANCE OF MICROGLIA IN BRAIN HEALTH AND DISEASE}

\section{Development}

Microglia represent 5 to $15 \%$ of the adult CNS cell population and constitute the largest population of immune cells in the brain. They play a major role in the development of the CNS, maintain homeostasis within the healthy brain, and can initiate, propagate, and/or resolve inflammatory responses. To understand how these cells are capable of such feats, it is important to understand the origin and cell lineage of microglia. Microglia originate from yolk sac erythromyeloid precursor cells in mice at embryonic day 7.5 (E7.5) (Alliot et al., 1991). These cells infiltrate both the neuro-epithelium and cephalic mesenchyme at days E8.5/E9.0 and undergo dramatic expansion until the second postnatal week (Alliot et al., 1991, 1999; Tambuyzer et al., 2009; Ginhoux et al., 2010; Mizutani et al., 2012; Crotti and Ransohoff, 2016). Infiltration into the CNS is not uniform, and location-dependent heterogeneity in density, morphology, and gene expression is observed (Lawson et al., 1990). Using scRNA-seq in conjunction with RNA immunohistochemistry (IHC) in situ across developmental time points, microglial heterogeneity within parenchyma, (PAM), and meninges, border-associated microglia (BAM), is now mapped into specific subpopulations by gene cluster (Goldmann et al., 2016; Prinz et al., 2017; Li et al., 2019; Ochocka et al., 2019; 
Van Hove et al., 2019). Although microglial ontogeny is mostly preserved across subpopulations, the region of the brain in which the microglia reside significantly affects the phenotype and response to their microenvironment (Grabert et al., 2016). At E9.5, yolk sac progenitors develop into embryonic microglia (Rosenbauer and Tenen, 2007). These embryonic microglia are a distinct tissue specific macrophage population, which is different from circulating myeloid cells. Specifically, erythromyeloid precursor cells develop into $\mathrm{CD}_{4} 5^{+}, \mathrm{c}^{-\mathrm{kit}^{l o}}, \mathrm{C}-\mathrm{X} 3-\mathrm{C}$ Motif Chemokine Receptor 1 (CX3CR1)immature cells, when in turn mature into $\mathrm{CD}_{4}{ }^{+}, \mathrm{c}^{-} \mathrm{kit}^{-}, \mathrm{CX} 3 \mathrm{CR} 1^{+}$, which invade the brain using specific metalloproteases (Kierdorf et al., 2013). Following infiltration, factors such as Transforming growth factor $\beta$ (TGF $\beta$ ), IL-34 and Colony stimulating factor 1 receptor (CSF$1 \mathrm{R})$ are necessary for the terminal differentiation of resident microglia (Butovsky et al., 2014). Although it remains somewhat controversial, recent studies suggest that in addition to canonical, non-Hoxb8 expressing microglia that infiltrate the brain at E9.5, there is a "second wave" of yolk-sac progenitors that are greatly expanded in the fetal liver prior to infiltrating the brain at E12.5 (De et al., 2018). Importantly, this second wave of cells may give rise to microglia-like cells, with distinct characteristics such as expression of Membrane-spanning 4-domains subfamily A member 7 (Ms4a7) and ApoE (Bennett and Bennett, 2020).

At E11/E16, neural stem cells (NSCs) at the dorsoventral boundary begin a massive expansion and differentiate into neural progenitor cells (NPCs), oligodendrocyte precursor cells (OPCs), and mature oligodendrocytes (OLs) (Naruse et al., 2017). Over half of these mature OLs eventually undergo apoptosis (Barres et al., 1992). Many of these mature apoptotic OLs are phagocytosed by an early phagocytic subset of PAM (Li et al., 2019), highlighting the role of microglia in primary myelination. In addition, a subtype of microglia associated with white matter and axon tracts express high levels of LPL and Secreted Phosphoprotein 1 (Spp1) (Hammond et al., 2019), which could potentially facilitate clearance of myelin-derived lipid debris. Chemokines such as chemokine (C-X-C motif) ligand 1 (CXCL1), expressed in high quantities by neurons, stimulate microglial migration (Harrison et al., 1998; Jakovcevski et al., 2009), potentially to sites with active myelinogenesis. It is thought that microglia regulate primary myelinogenesis through a critical source of neuroprotective Insulin growth factor 1 (IGF1), known to regulate OL function (Wlodarczyk et al., 2017). Mature OLs are lipid-rich, requiring cholesterol and both essential and non-essential fatty acids (FAs) to effectively produce myelin (Mathews and Appel, 2016; Dimas et al., 2019). Although astrocytes provide OLs with cholesterol via effluxed lipoprotein particles, OLs can also produce cholesterol de novo via Phosphoinositide 3-kinase/Protein kinase $\mathrm{B} /$ mammalian target of rapamycin (PI3K)/(Akt)/(mTOR) signaling (Chen et al., 2013; Mathews and Appel, 2016). The precise role of microglia in OL lipid metabolism is unclear, however, studies outlined above suggest a key role in the phagocytosis of lipid-rich debris from myelin and apoptosed OPCs and OLs.

While recent studies have shown that microglia are extremely diverse during early development, it is clear that they become less heterogeneous during adulthood (Hammond et al., 2019).
Nonetheless, the dynamic transcriptional signatures of microglia exposed to injury, aging or disease, suggest that the adult microglia are readily poised to adapt to challenges in the local environment.

\section{Synaptic Pruning}

It has been previously observed in healthy brain that so-called "resting microglia" are in fact very active. They are highly mobile and take, on average, a 5 min sample of one neuronal synapse per hour (Davalos et al., 2005; Nimmerjahn et al., 2005; Wake et al., 2009). Additionally, synapses in ischemic areas are turned over following microglial detection (Wake et al., 2009). This sampling of synapses hints at the possibility that microglia are involved in the crucial process of synaptic pruning. The expression of the chemokine fractalkine (CX3CL1), a strong microglial chemoattractant and whose receptor in the CNS is expressed solely in microglia, is up-regulated in neurons during development (Harrison et al., 1998; Jung et al., 2000; Cardona et al., 2006; Liang et al., 2009). Through GFP-labeling of microglia and immunohistochemistry against postsynaptic density protein 95 (PSD95), a marker of postsynaptic density, stimulated emission depletion (STED) microscopy revealed colocalization of PSD95 and GFP. Moreover, electron microscopy of these microglia show the presence of both clathrin-coated and non-clathrin-coated vesicles containing PSD95 as well as Synaptosome Associated Protein 25 (SNAP25), a marker of presynaptic density (Paolicelli et al., 2011). These data strongly suggest that microglia are actively involved in synaptic pruning. A closer look at hippocampal synapses via light sheet fluorescence microscopy and 3D ultrastructural characterization using Correlative light and electron microscopy (CLEM) found that microglia do not directly phagocytose entire synapses (Weinhard et al., 2018). Instead, they trogocytose-selective partial phagocytosis - the membranes of presynaptic boutons and axons without any evidence for elimination of dendritic spines (Weinhard et al., 2018). The exact mechanism of trogocytosis has yet to be elucidated.

\section{Phagocytosis}

Microglia play an important role as the immune effector cells of the central nervous system. In line with this role, microglia express many cell surface factors that are also expressed by peripheral myeloid cells and macrophages such as integrin's, toll-like receptors, scavenger receptors, and TREM2 (Aldana, 2019). Although these similarities have made the isolated study of microglia somewhat challenging, there has been a concerted effort to define the characteristics that set microglia apart from other myeloid cells. For example, while microglia and bone marrow derived macrophages both express CD11B and CD45 (although high for macrophages and low for microglia), microglia express higher levels of Transmembrane Protein 119 (Tmem119), CX3CR1 and Solute Carrier Family 2 Member 5 (Slc2a5) (Haage et al., 2019). It is likely that this differential expression represents the homeostatic functions that are specific to microglia and not peripheral cells such as synaptic pruning, neuronal survival, and synaptogenesis (Bilimoria and Stevens, 2015). Such neuroprotective functions are largely due to the 
phagocytic nature of microglia (Janda et al., 2018). Microglia are highly efficient phagocytes that remove apoptotic or necrotic cells (Green et al., 2016), and unfolded proteins such as amyloid beta $(A \beta)$ or neuromelanin. Engulfment of myelin debris is also a key function of microglial sub-populations associated with remyelination and repair (Olah et al., 2012). Furthermore, microglia are an important part of the innate immune system and are activated in response to infections in order to directly phagocytose potentially pathogenic microorganisms (Nau et al., 2014). Microglial phagocytosis initiates the adaptive arm of the immune system via antigen presentation (Litman et al., 2005). Overall, the immune functions of microglia showcase their plasticity and ability to respond to a wide variety of stimuli. However, the response can sometimes become chronic and maladaptive. This prolonged microglial activation is a salient feature of many NDs such as AD, Parkinson's disease and MS.

\section{Disease}

\section{Metabolic Disorders}

Metabolic disorders such as obesity have implicated in the pathological activation of microglia largely due to an increase in systemic inflammation. For example, the leptin deficient mouse model of type 2 diabetes $(\mathrm{db} / \mathrm{db})$, shows increased inflammatory chemokine and cytokine expression in the CNS (Kumar et al., 2014). In addition, several studies have shown that exposure to a high-fat diet (HFD) can increase microglial activation, even without peripheral inflammation (Ziko et al., 2014; Kang et al., 2016). In fact, recent work as shown that only 3 days of HFD exposure is sufficient to promote gliosis in the hypothalamus (Thaler et al., 2012). Since microglia are seeded developmentally and are particularly long-lived, they are a potential conduit for the transmission of "developmentally programmed" dietary exposures. In support of this hypothesis, microglia in the paraventricular nucleus of the hypothalamus can be programmed to an active state following HFD exposure during very early life; a phenotype that persists into adulthood (Morari et al., 2014). It is likely that circulating inflammatory mediators and lipids and lipoproteins have greater penetrance to the hypothalamus than other regions of the brain, which may explain the rapid metabolic polarization of microglia in this region.

\section{Schizophrenia}

Dysregulation of synaptic pruning has been increasingly implicated in the pathophysiology of both NDs and psychiatric disorders. In schizophrenia (SZ), patients have decreased gray matter thickness and reduced overall brain volume (Ziermans et al., 2012; Cannon et al., 2015). This is correlative with a decrease in synaptic density (Glantz and Lewis, 2000; Glausier and Lewis, 2013). To study this phenomenon, peripheral monocytes were induced into a validated microglia-like (iMG) cell culture model (Sellgren et al., 2019). Interestingly, iMGs from patients with SZ that are co-cultured with neurons display elevated internalization of PSD95 and SNAP25 as well as a utilization of the complement system, suggesting that microglial synaptic pruning is increased in patients with ongoing SZ (Sellgren et al., 2019). Microglia-mediated degradation of synapses is also seen in AD (Lacor et al., 2004; Chu et al., 2010;
Schafer et al., 2012; Bialas and Stevens, 2013; Sekar et al., 2016; Panayiotou et al., 2017; Shi et al., 2017).

\section{Alzheimer's Disease}

Alzheimer's Disease is the most common cause of dementia in the elderly, characterized by gradual memory loss and cognitive decline. The first phase of the disease involves the subclinical, gradual buildup of extra-cellular monomeric $\mathrm{A} \beta$ $(\mathrm{mA} \beta)$ that coalesces into oligomers and eventually larger amyloid fibrils/plaques. This leads to the clinically significant second phase of the disease, involving the formation of hyperphosphorylated tau neurofibrillary tangles that are associated and the destruction of neurons; the hallmark of AD. Although cognitive decline occurs most drastically during the second phase, the gradual phase has also been implicated in the deterioration of synaptic density as a result of increased microglia-mediated synapse loss (Carroll, 2004; Gasque, 2004; Reichwald et al., 2009; Ransohoff, 2016). This is primarily facilitated by fibrillar $\mathrm{A} \beta$ (fA $\beta$ ) and oligomeric $\mathrm{A} \beta$ (oA $\beta$ ) aggregation onto neuronal post-synaptic terminals leading to complement deposition, microglial activation following synapse elimination and neural network dysfunction (Carroll, 2004; Gasque, 2004; Reichwald et al., 2009; Pan et al., 2011; Ransohoff, 2016). C3 receptor (CD11b/CD18) wielding microglia are the key mediators of $\mathrm{A} \beta$ clearance and show a differential phagocytic response depending on the morphology of the encountered A $\beta$ (Carroll, 2004; Gasque, 2004; Reichwald et al., 2009; Ransohoff, 2016). Microglial exposure to fA $\beta$ induces a classical phagocytic response, which may help to facilitate the removal of $\mathrm{fA} \beta$ and prevent plaque formation (Pan et al., 2011). In support, recent scRNA-seq studies in the 5XFAD model of $\mathrm{AD}$ have shown a profound increase in DAM, which have elevated expression of factors involved in lipid uptake and phagocytosis, likely in an attempt to clear $\mathrm{fA} \beta$ at later stages of the disease (Keren-Shaul et al., 2017). Although the role of microglia in $\mathrm{AD}$ pathogenesis is a research-intensive area, whether specific microglial subpopulations contribute or prevent $\mathrm{AD}$ progression remains to be empirically determined.

The clearance of $A \beta$ and its tendency to aggregate can be significantly altered by lipid and lipoprotein metabolism (see Figure 1A). Interestingly, Alois Alzheimer originally described an increased quantity of "lipoid granules" in the $\mathrm{AD}$ brain as a pathological hallmark, suggesting that irregular lipid and lipoprotein metabolism may be a driving factor (Foley, 2010). Increased free-cholesterol containing lipid rafts (Riddell et al., 2001; Ehehalt et al., 2003; Hattori et al., 2006; Marquer et al., 2011), which have been shown to increase A $\beta$ aggregation (Wisniewski et al., 1994; Bhattacharyya and Kovacs, 2010; Bryleva et al., 2010) may be a potential underlying mechanism. Additionally, ApoE, the most abundant apolipoprotein in the CNS, is also known to modulate $\mathrm{A} \beta$ aggregation in an isoform dependent manner (LaDu et al., 1994). Furthermore, gangliosides (which contain ceramide) within $A \beta$ aggregates may contribute to the persistence of amyloid plaques by evading microglial detection via their interaction with sialic acid-binding Ig-like lectin (siglec-11), 


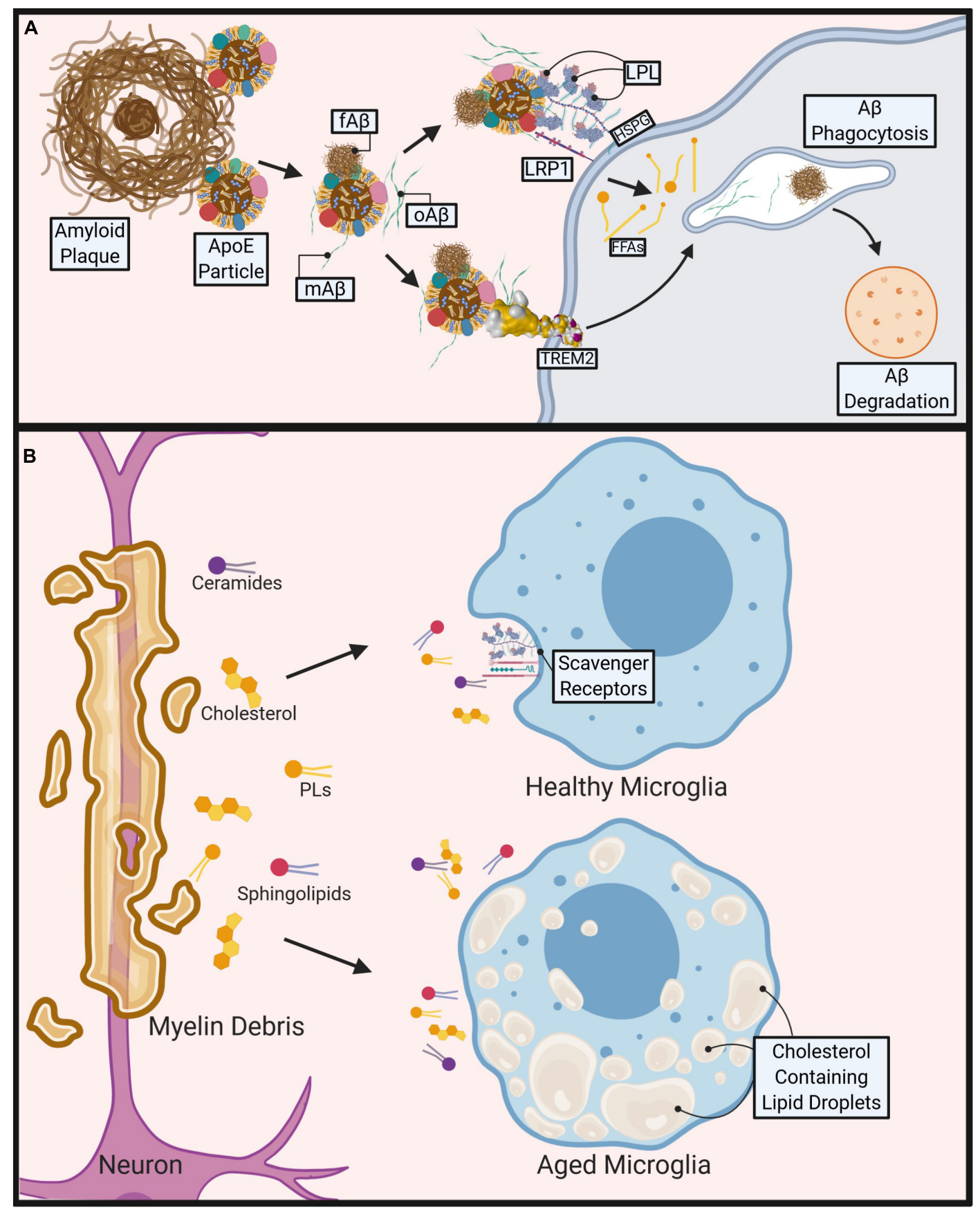

FIGURE 1 | Role of microglial lipid metabolism in the pathogenesis of Alzheimer's disease (AD) and Multiple Sclerosis (MS). (A) During AD, Apolipoprotein E4 (ApoE4) containing particles are thought to cause polymerization of Amyloid-Beta ( $A \beta$ ) contributing to fibrillar $A \beta$ ( $A A \beta$ ), plaque formation and $A D$ pathogenesis. $A \beta$ phagocytosis/clearance may prevent plaque formation. TREM2 binds to oligomeric $A \beta(O A B)$ and may bind to $A p o E$ containing lipoprotein particle-bound monomeric $A \beta(m A \beta)$ and $f A \beta$ to facilitate phagocytosis and degradation of $A \beta$. Lipoprotein Receptor-like protein 1 (LRP1) and Lipoprotein Lipase (LPL) tethered to the microglial cell surface via Heparan sulfate proteoglycans (HSPGs) may also bind to $A \beta$ directly or via an interaction with $A \beta$-bound ApoE containing lipoproteins, to facilitate $A \beta$ uptake and degradation. (B) During MS, demyelination causes release of myelin-derived lipids such as ceramides, cholesterol, phospholipids (PL), and sphingolipids. Healthy microglia clear lipids via cell surface scavenger receptors (e.g., LRP1, LPL). However, aged, or pro-inflammatory microglia accumulate cholesterol and other neutral lipids, which impairs their ability to phagocytose myelin debris, leading to reduced and abnormal remyelination. Created with Biorender.com.

a negative immune receptor (Salminen and Kaarniranta, 2009). There is an increasing focus toward microglia-mediated lipid and lipoprotein metabolism in the brain and their effects on systemic and neuroinflammatory diseases (Di Paolo and Kim, 2011; Bruce et al., 2017; Reale and Sanchez-Ramon, 2017; Xicoy et al., 2019). 


\section{Multiple Sclerosis}

In progressive forms of MS, the progression of chronically active demyelination lesions leads to worsening disability (Absinta et al., 2019). Within these lesions, myelin, which is composed mostly of lipid, is destroyed by microglia and lipid-laden infiltrating macrophages amongst other immune cells (Raine et al., 1981). It is here that lipid metabolism is most crucial in the modulation of disease. The presence of myelin debris itself is known to cause a sustained inflammatory response (Clarner et al., 2012; Cantuti-Castelvetri et al., 2018; Kopper and Gensel, 2018). Additionally, cholesterol-breakdown products are significantly higher within the brain and CSF of these patients. 7ketocholesterol $(7 \mathrm{KC})$ is among these products and is associated with mitochondrial dysfunction and attenuated lipid processing through FA oxidation (FAO) in OLs (Diestel et al., 2003; Leoni et al., 2017). In microglia, $7 \mathrm{KC}$ rapidly enters the nucleus and activates poly (ADP-ribose)-polymerase (PARP)-1, which induces a pro-inflammatory phenotype (Diestel et al., 2003; Kauppinen and Swanson, 2005). In some ways this is helpful, as activated microglia are more mobile and significantly increase their phagocytic activity in order to clear debris effectively (Fu et al., 2014). However, a recent study has shown that as phagocytic microglia age they lose the capacity to effectively efflux cholesterol, form intracellular cholesterol crystals, and propagate a maladaptive pro-inflammatory response (CantutiCastelvetri et al., 2018) (see Figure 1B). In contrast, omega-3 (n-3) polyunsaturated FAs (PUFAs) have been shown to reduce inflammation and induce an anti-inflammatory phenotype within microglia (Hopperton et al., 2016; Chen et al., 2018; Layé et al., 2018). Similarly, an increased intake of total dietary PUFAs is associated with a reduced risk of developing MS (Bjornevik et al., 2017). In addition, we have previously shown that LPL, the rate-limiting enzyme in the hydrolysis of triglyceride (TG) rich lipoproteins, is associated with an anti-inflammatory microglial phenotype due to its complex role in lipid uptake and metabolic reprogramming (Bruce et al., 2018).

\section{METABOLIC REPROGRAMMING IN MACROPHAGES AND MICROGLIA}

It is well established that macrophages change their metabolic profile to meet the increased bioenergetic demands of activation. Although a similar "metabolic reprogramming" also occurs in microglia, our understanding of this process is less well understood. For this reason, it is useful to review the literature describing the interaction between metabolism and inflammation in macrophages to serve as a relative model for microglia. When macrophages are homeostatic, or quiescent, they catabolize various substrates (glucose, amino acids, fatty acids) to be utilized in the TCA cycle to produce electrons (carried in the form of NADH/FADH2). These electrons are then used during mitochondrial oxidative phosphorylation (OXPHOS) to drive adenosine triphosphate (ATP) production (Mehta et al., 2017). In contrast, pro-inflammatory macrophages show a metabolic shift toward glycolysis and away from OXPHOS (Van den Bossche and Saraber, 2018). The metabolic preference for energy derived from glycolysis over OXPHOS in normoxic conditions is reminiscent of the metabolic profile of many types of tumors. In these tumor cells, glycolysis predominates despite sufficient oxygen for oxidative metabolism to proceed; a phenomenon first described by Otto Warburg in 1927, thus termed the "Warburg effect" (Warburg et al., 1927). In macrophages, metabolic polarization to a Warburg-like metabolism can follow exposure to a range of stimuli, including LPS, the toll-like receptor (TLR) 3 ligand poly(I:C), and type 1 interferon (IFN) (Kelly and O'Neill, 2015). It has been suggested that activated macrophages shift toward glycolytic metabolism to preserve the macromolecules that may be needed to synthesize new proteins required for a given activation state (Rambold and Pearce, 2018). For example, glucose metabolism feeds the pentose phosphate pathway (PPP), which increases the production of purines and pyrimidines, important for biosynthesis in the activated cell. Increased flux through the PPP also provides NADPH for NADPH oxidase, which generates reactive oxygen species (ROS) that can be used as an anti-bacterial mechanism (West et al., 2011). It is also thought that the increase in glycolysis generates ATP quickly, and although inefficient in comparison to oxidative metabolism, provides the necessary energy to support cell activation in the shortest time frame. Importantly, recent studies using microglia isolated from 5XFAD mice have shown similar impairments in metabolism including shifts toward glycolysis, which can be reversed following INF- $\gamma$ treatment (Baik et al., 2019). There are a number of mechanisms thought to contribute to the metabolic reprogramming of macrophages and microglia, although here we will focus on those involving alterations in lipid metabolism, there are several reviews that discuss the mechanisms in more detail (Kelly and O’Neill, 2015; Langston et al., 2017).

Macrophage lipid metabolism is profoundly altered during polarization. For example, following macrophage activation, the TCA cycle is interrupted and acetyl-CoA is shunted to the synthesis of lipid precursors for inflammatory mediators (Jha et al., 2015). Specifically, following intraperitoneal (IP) injection of LPS or zymosan (a ligand found on surface of yeast) there is a dramatic increase in the synthesis of cholesterol ester (CE) and FAs in peritoneal macrophages (Posokhova et al., 2008). Furthermore, activated macrophages take up significantly more native and acetylated low-densitylipoproteins (LDL), which contributes to CE synthesis, whereas non-acetylated LDL uptake is inhibitory to CE synthesis in control cells (Wang et al., 2007). In addition, LPS treatment leads to TG accumulation in macrophages, which is coupled with decreased FAO and TG lipolysis, without altered expression of intracellular lipases (Adipose triglyceride lipase [ATGL] and hormone sensitive lipase [HSL]) (Feingold et al., 2012). In contrast, 'alternative activation' of macrophages is associated with increased FAO, raising the possibility that controlling fatty acid metabolism may attenuate inappropriate activation (Namgaladze and Brune, 2016). Several targets have been identified that may control FA metabolism in the context of inflammation, with a particular emphasis on mitochondrial FAO. For example, promoting mitochondrial $\beta$-oxidation has been shown to attenuate endoplasmic reticulum (ER) stress that can be induced by the saturated fatty acid (SFA) palmitate 
(C16:0) (Namgaladze et al., 2014). Conversely, knockdown, or pharmacological inhibition of mitochondrial FAO leads to exacerbated ER stress and inflammation in response to palmitate (Namgaladze et al., 2014). In support, over expression of a mutant from of Carnitine Palmitoyltransferase 1A (CPT1A) that is insensitive to malonyl-CoA inhibition (and thus shows enhanced mitochondrial FAO) prevents palmitate-induced inflammation, ER stress, and oxidative damage (Malandrino et al., 2015). While there may be deleterious effects of increased FAO, particularly in tissue-specific or hypoxic conditions, overall it has been suggested that a major consequence of increased macrophage FAO is a diversion of fatty acid flux away from the formation of FA metabolites that cause ER stress and are precursors for inflammatory mediators.

Until recently, our understanding of how microglial metabolism relates to activation and function has been somewhat extrapolated from the peripheral macrophage literature. However, recent scRNA-seq studies mentioned above have highlighted that microglial metabolism may be similarly altered in response to changing bioenergetics during the development of NDs. For example, several studies have highlighted the need for increased expression of genes associated with lipid metabolism (e.g., TREM2, LPL and ApoE) during development, damage and disease (Keren-Shaul et al., 2017; Hammond et al., 2019). While this suggests a need for increased FAO in these activation states, further studies are needed to empirically define the role of these factors in microglial lipid metabolism and inflammation, particularly in the context of ND. It is important to point out that while the metabolism of microglia may be similar to macrophages in many aspects, it is likely that access to limited metabolic substrates in the CNS (e.g., no very-low-density-lipoproteins [VLDL] or LDL) has led to the development of somewhat distinct metabolic profiles, that have not yet been clearly defined.

\section{FATTY ACID METABOLISM IN MICROGLIA}

\section{Overview}

The brain's dry weight is made up of about $50 \%$ lipid, most of which is comprised of myelin enriched white matter (Hamilton et al., 2007). Myelin is a specialized OL membrane that contains $\sim 43 \%$ phospholipid (PL), 28\% glycosphingolipid, and 28\% cholesterol of its total lipid content (Morell, 1999). PLs are derived from FAs in a biosynthetic pathway that is initiated by fatty acyl-CoA synthase, which combines a FA with acylcoenzyme A (acyl-CoA) using ATP to create fatty acyl-CoA (Yamashita et al., 2014). Glycerol-3-phosphate acyltransferase (GPAT) adds a glycerol backbone, creating a single-tailed lysophosphatidic acid (LPA) (Yamashita et al., 2014). CoA replenishes it's acyl group from acyl-carnitine via the carnitine transport system within the mitochondria (Yamashita et al., 2014). Within the ER, LPA is further modulated to give rise to TGs, diacylglycerols (DAGs), and PLs (Yamashita et al., 2014). These PLs are transported through the Golgi, to other organelles, and to the polymorphic cell surface along with proteins, cholesterol, and other FA containing glycolipids (GLs). PLs are important for providing membrane integrity, lipid raft formation, signal transduction, providing curvature to the cell membrane, vesicle formation, apoptosis, and in the production of pro- or anti-inflammatory mediators. Moreover, they are becoming increasingly implicated in the pathophysiology of NDs.

\section{Phospholipids}

Phospholipids constitute $45 \%$ of the total dry weight of the brain, and are key to several pathways, such as the synthesis and turnover of neuronal and glial membranes and signaling. Since these processes are critical to CNS health, it is not surprising that there are substantial energy demands associated with PL metabolism, with $5 \%$ of the brains ATP being used for the turnover of 1,2-diacyl type PLs (Purdon et al., 2002). A number of reports have suggested that FAs and PLs are increased during pathological conditions such as inflammation, hypoxia and ischemia, and it is likely that this represents release of FAs from brain PLs, or indeed PLs from vital brain structures. For example, in individuals with PD, total serum PL levels are increased without any signs of cognitive impairment and positively correlate with the progression of their cognitive impairment (Li et al., 2015). This finding suggests that increased PL levels could be an early marker for PD onset and disease progression. Furthermore, the enzymes involved in synthesizing phosphatidylserine (PS), phosphatidylcholine (PC) and phosphatidylethanolamine (PE) are increased in the substantia nigra of PD patients (Ross et al., 2001). The increase in total serum PL may indicate cell membrane damage in the substantia nigra, which triggers a compensatory increase in PS, $\mathrm{PE}$, and PC synthesis. Interestingly, while total serum PL is increased, PS 40:4 is decreased (Zhang et al., 2017). In nonapoptotic cells, PS is asymmetrically present within the inner layer of the cell membrane. However, in PD, apoptotic neurons extrovert PS to the surface of the cell membrane, via inactivated flippase and activated scramblase enzymes, to signal phagocytes to remove the cell (Wei et al., 2013). Microglia are known to target these apoptotic cells via expression of PS-specific receptors (Fadok et al., 2000). In recent years several microglia-derived factors that participate in PS-stimulated phagocytosis have been identified. For example, Milk fat globule epidermal growth factor8 (MFG-E8) is secreted by microglia and links exposed neuronal PS to vitronectin receptors, which modify the actin cytoskeleton to stimulate microglial phagocytosis of dying neurons (Fricker et al., 2012). Therefore, it is plausible that activated microglia may have increased uptake of PS during inflammation, which could potentially drive decreased serum levels observed in PD. It is also plausible that elevated serum PL, could indicate defective microglial phagocytosis.

\section{PUFAs}

Polyunsaturated fatty acids have more than one carbon-carbon double bond within the FA hydrocarbon chain. n-3 PUFAs are characterized by a double bond three atoms away from the $\omega$-methyl group. While mammals cannot synthesize n3 PUFAs, they are available from the diet in the form of essential FAs, such as alpha-linolenic acid (ALA). n-3 PUFAs 
are associated with a range of benefits: from preventing age related cognitive decline to preventing metabolic syndrome and improving cardiovascular risk factors (Schwinkendorf et al., 2011; Layé et al., 2015; Trépanier et al., 2016). In contrast, omega6 (n-6) PUFAs, which have their last double bond 6 atoms away from the terminal methyl group, are generally associated with the worsening of many chronic diseases such as obesity, neuroinflammatory diseases, and asthma (Simopoulos, 2016; Brigham et al., 2019). Within the cell membrane, the ratio of n-3 to n-6 PUFAs can influence cell functions (Hennig and Watkins, 1989; Schwinkendorf et al., 2011; Simopoulos, 2016). Both n-3 and n-6 PUFAs are synthesized using the same elongases and desaturases, so they must compete for their creation and integration into the cell membrane (Simopoulos, 2011). PUFAs are abundant within the CNS, making up 30\% of the brain's FAs; n-3 PUFAs account for a third of these (Hamilton et al., 2007). The distribution of PUFAs isn't homogenous across lipid class (Simopoulos, 2008). For example, TGs and CEs contain eicosatetraenoic acid (EPA), 20:5, and ALA, whereas ALA is scarcely present in PLs, which contain a high concentration of docosahexaenoic acid (DHA), 22:6, and EPA (Simopoulos, 2008). PUFAs are also heterogeneously located across different brain regions. About $30 \%$ of all fatty acids in the outer segment membrane of retinal photoreceptors are n-3 PUFAs (Bazan et al., 1990). Consequently, a diet rich in n-3 PUFAs significantly slows the decline in visual acuity in patients with retinitis pigmentosa (Berson et al., 2012). In addition, the most prevalent n-3 PUFA in the body, DHA, is found at concentrations that are several hundred-fold higher than EPA in the brain and retina (Arterburn et al., 2006). This may be caused by the brain's preferential usage of EPA as an energy source that is rapidly catabolized via FAO (Chen and Bazinet, 2015). Importantly, DHA cannot be synthesized de novo in the brain, and until fairly recently, the mechanism of long chain (LC)-FA transportation across the blood brain barrier (BBB) has remained unknown. However, recent studies have shown that a member of the Major Facilitator Superfamily Domain Containing 2A (Mfsd2a) can transport DHA, in the form of lysophosphatidylcholine (LPC) (Nguyen et al., 2014). In support, Mfsd2a-knockout mice show markedly reduced levels of DHA in the brain, accompanied by neuronal loss and cognitive deficits (Nguyen et al., 2014). Although initially studies report the expression of Mfsd2a largely in the endothelial cell, subsequent studies have also shown that Mfsd2a is present, albeit at a lower level in OLs, OPCs and astrocytes (Chan et al., 2018). Moreover, increased numbers of activated microglia are observed in the subretinal space of Mfsd2a knockout mice compared to wild-type controls (Wong et al., 2016).

Omega-3 and omega-6 PUFA containing PLs bring about dichotomous secondary mediators when turned over at the level of the cell membrane. The phospholipase $\mathrm{A}_{2}\left(\mathrm{PLA}_{2}\right)$ superfamily of enzymes, hydrolyze FAs from the sn-2 position of membrane PLs, and contain 15 groups that differ in their ability to recognize and respond to various PL substrates. Among these groups, cytosolic group IV of $\mathrm{PLA}_{2}\left(\mathrm{cPLA}_{2}\right)$ releases arachidonic acid (AA), whereas calcium dependent group VI $\mathrm{PLA}_{2}$ releases DHA (Jenkins et al., 2004). Typically, free DHA is enzymatically metabolized by cyclooxygenase 2 (COX2) and lipoxygenases (LOXs) into resolvins, protectins, and maresins, which are known to be potent anti-inflammatory specialized proresolving mediators (SPMs) (Serhan, 2014; Calder, 2015). Free AA, however, is metabolized into prostaglandins, prostacyclins, thromboxanes, and leukotrienes. With a few exceptions, these AA derived mediators are known to produce potent proinflammatory effects (Lukiw and Bazan, 2000). Positron emission tomography (PET) has been used to study AA incorporation into the brain (Robinson et al., 1992; Rapoport, 1999), and made important contributions to our understanding of AA turnover and metabolism in various contexts. For example, PET imaging has revealed that AA uptake is elevated in widespread cortical regions in patients with $\mathrm{AD}$ compared to healthy controls, which is consistent with the notion that elevated AA is a marker for neuroinflammation (Esposito et al., 2008).

Within microglia, the downstream effects of n-3 PUFA metabolism has been observed to be beneficial in the context of neuroinflammatory diseases. n-3 PUFA supplementation attenuates activation by deacetylation of the High Mobility Group Box 1/Nuclear Factor kappa-light-chain-enhancer of activated $\mathrm{B}$ cells (HMGB1)/(NF-kB) pathway in a traumatic brain injury (TBI) mouse model (Chen et al., 2018). This in turn leads to decreased inflammatory markers and neuroprotective effects following injury (Chen et al., 2018). Although n-6 PUFAs are generally considered pro-inflammatory, this is not the case for all n-6 PUFAs. For example, supplementation with palmitic acid, a 16-C SFA, can activate microglia ( Tu et al., 2019). However, supplementation with n-6 linoleic acid (LA) is sufficient to reverse this activation (Tu et al., 2019). In addition to microglial polarization, a growing body of evidence suggests that different FA classes uniquely alter energy metabolism irrespective of activation status (Button et al., 2014; Chausse et al., 2019). In a study with real-time metabolic measurements coupled with lipidomic analysis, both oleate, a monounsaturated FA (MUFA), and palmitate, a SFA, promoted oxidative metabolism whereas LPS increased glycolysis (Chausse et al., 2019). Although both FAs promoted OXPHOS, oleate treatment increased CD36 abundance (an important scavenger receptor and LC-FA transporter), as well as PUFA-containing TGs, while palmitate incorporated more PUFAs into PLs (Chausse et al., 2019). It is likely that SFA-induced incorporation of PUFAs into PLs contributes to inflammation since PUFAs are more readily peroxidized (Chausse et al., 2019). It is also of note that LPS activation can decrease MUFAs while increasing SFA concentrations, both of which can induce a pro-inflammatory effect (Button et al., 2014). Further studies using metabolomic and lipidomic analysis need to be conducted to fully elucidate the metabolic alterations that take place in microglia in response to different FAs.

Omega-3 PUFA supplementation has also been shown to enhance microglial phagocytosis of myelin (Chen et al., 2014). Several studies have demonstrated that increasing the $n-3$ to $n-6$ ratio in fat- 1 mice, which express an n-3 FA desaturase that converts n-6 PUFAs to n-3 PUFAs, has beneficial results on the inflammatory status of microglia (Ma et al., 2006; Kang, 2007; Zhang et al., 2017). A recent 
study used the cuprizone-model of demyelination and remyelination to compare fat- 1 mice to WT (Siegert et al., 2017). The CNS lipid profile revealed increased remyelination in fat-1 mice as well as increased EPA levels and EPA metabolites, such as 18-hydroxyeicosapentaenoic acid (18HEPE) (Siegert et al., 2017). As previously stated, EPA has a relatively low quantity in the CNS lipid profile, and is rapidly utilized as a carbon source in FAO (Chen and Bazinet, 2015). Taken together, perhaps the energy provided from FAO of EPA is critical in fueling phagocytosis of myelin and production of secondary mediators that further aid in the process of remyelination. It is also possible that EPA maintains oxidative metabolism and prevents the shift toward glycolysis that occurs during pro-inflammatory and metabolic reprogramming in microglia.

In addition to fueling oxidative metabolism, PUFAs are also agonists for nuclear receptors with important roles in microglial activation and function. For example, the peroxisome proliferator activated receptors (PPARs) are a family of nuclear receptors with key roles in lipid and glucose metabolism, inflammation, and proliferation. All three PPAR isoforms $(\alpha$, $\beta / \delta$ and $\gamma$ ) are activated by naturally occurring FAs such as $\mathrm{AA}$ and EPA. Moreover, the downstream metabolite of AA, 15-deoxy- $\Delta^{12-14}-\mathrm{PGJ}_{2}$ (e.g., 15d-PGJ $)_{2}$ ), was the first reported endogenous ligand for PPAR- $\gamma$ (Forman et al., 1995; Kliewer et al., 1995). Differences in the concentration required to activate PPAR- $\gamma$ in vitro, and the concentrations naturally present, have called the physiological relevance of $15 \mathrm{~d}-\mathrm{PGJ} \mathrm{J}_{2}$ into question. Nonetheless, PPAR- $\gamma$ is expressed in primary microglial cells, and its expression is enhanced following $15 \mathrm{~d}-\mathrm{PGJ}_{2}$ supplementation (Bernardo et al., 2000). Interestingly LPS-stimulated primary microglia synthesize large amounts of $15 \mathrm{~d}-\mathrm{PGJ}_{2}$, which is able to downregulate microglial activation through both PPAR- $\gamma$-dependent and independent mechanisms (Bernardo et al., 2003). Since these initial findings, several studies have now shown that natural and synthetic PPAR- $\gamma$ agonists can down-regulate surface antigens and inflammatory mediators (Fumagalli et al., 2018), while increasing expression of anti-inflammatory factors such as Arginase 1 (ARG1) and IL-4 (Song et al., 2016). It is likely that the pivotal role of PPAR- $\gamma$ in microglial activation lies with its role in metabolic reprogramming. PPAR- $\gamma$ signaling directs microglia toward protective functions, such as increased FAO and OXPHOS (Fumagalli et al., 2018). Therefore, PPAR- $\gamma$ agonists have been extensively studied as potential therapeutics to ameliorate aberrant microglial activation during $\mathrm{ND}$, with mixed results.

\section{FAs in Oxidative Metabolism}

Although the brain is only $2 \%$ of the total body mass, it utilizes $\sim 20 \%$ of the body's total ATP. While glucose is the primary source of energy for the brain, it has since been estimated that $20 \%$ of the brain's energy production stems from FAO (Ebert et al., 2003; Bélanger et al., 2011). FAs have also been shown to enter the mammalian brain (Dhopeshwarkar and Mead, 1970; Dhopeshwarkar et al., 1971; Taha et al., 2016). Recent evidence suggests that FA uptake by the brain is both a multifaceted and protein-mediated process (Murphy, 2017). It is likely that technical advances may lead to a deeper understanding of brain metabolism and may challenge the dogma of (largely) exclusive glucose utilization by the brain. In support, several studies have shown that FAs are oxidized in the brain during development and adulthood (Allweis et al., 1966; Warshaw and Terry, 1976). It is believed that FAO occurs predominantly in astrocytes (Ebert et al., 2003). However, since isolated neuronal and glial cells, and mitochondria from both, are able to use FAs to fuel oxidative metabolism (Chausse et al., 2019), it is not implausible that FAs provide an alternative source of energy to neurons and glia, either directly, or via astrocyte-derived lipoproteins (see Figure 2). Thus, a growing body of literature suggests that in addition to the role of lipids as components of myelin debris, or precursors to secondary messengers, FAs may also be an important driver of microglial oxidative metabolism. For example, many genes involved in fatty acid oxidation are expressed in microglia, but this expression is repressed following LPS or INF- $\gamma$ mediated activation (Mauerer et al., 2009). Importantly, this repression is damped by DHA supplementation (Mauerer et al., 2009).

IFN- $\beta$ is known to increase FAO and OXPHOS in macrophages ( $\mathrm{Wu}$ et al., 2016). Although an IFN- $\beta$-mediated increase in microglial FAO acid oxidation has not been empirically determined, IFN- $\beta$ is a well-known therapy for MS, and patients undergoing therapy show in an increase in FAO in peripheral myeloid cells (Croze et al., 2013). Moreover, recent studies have shown that supplementation with the IFN- $\beta$ in a rat model of $\mathrm{AD}$ is sufficient to ameliorate microglial activation, reduce ROS and lipid peroxidation, suggesting that IFN- $\beta$ helps polarize microglia to a phenotype that favors oxidative metabolism (Mudo et al., 2019). These studies highlight the therapeutic potential of IFN- $\beta$ and FAO to dampen aberrant microglial activation in ND.

Previously, we have shown microglia lacking LPL have significantly reduced FAO, suggesting that lipid uptake fuels lipid oxidation (Bruce et al., 2018). Conversely, L-carnitine supplementation has been shown to increase FAO and attenuate the LPS induced inflammatory response in microglia leading to improved cognitive and neuronal functions (Gill et al., 2018; Singh et al., 2018). Recent studies have also shown that both oleate and palmitate supplementation can sustain mitochondrial respiration in BV-2 microglia, while LPS treatment diminishes oxidative metabolism in favor of increased glycolysis (Chausse et al., 2019). Since LC-PUFAs are important for microglial function, it is likely that peroxisomal $\beta$-oxidation is vital to microglial metabolism. Indeed, studies in mice lacking the multi-functional protein-2 (MFP2), a pivotal enzyme in peroxisomal $\beta$-oxidation, become profoundly proinflammatory (Beckers et al., 2019). Although further studies are needed to identify the principle components of microglial oxidative metabolism, it is likely that FAO is necessary for physiologic energy production and maintenance of an antiinflammatory phenotype.

To utilize FAs for energy, they must first be taken up by the cell. FA transport proteins (FATPs), FA binding proteins (FABPs), and scavenger receptors that are categorized as class 


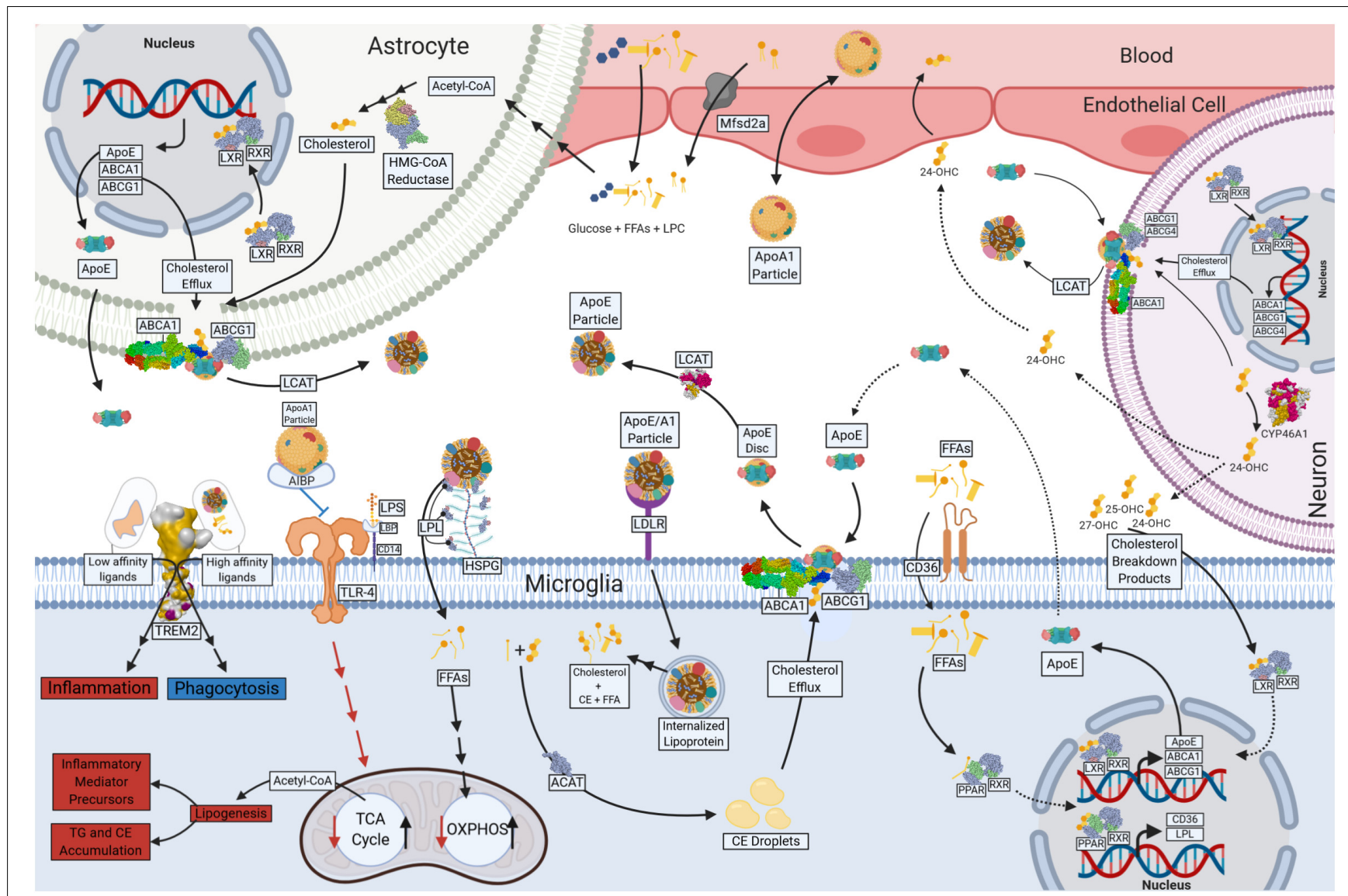

FIGURE 2 | Lipid and Lipoprotein metabolism in microglia. Free fatty acids (FFAs) and glucose cross the blood brain barrier (BBB) and provide energy substrates for neurons and glia. Apolipoprotein A1 (ApoA1) containing lipoproteins traverse the BBB and may play a role in lipid delivery to neurons and glia. Astrocytes are major source of CNS-derived lipoproteins and produce Apolipoprotein E (ApoE) containing lipoprotein particles, which is lipidated following ATP Binding Cassette Subfamily A Member 1 (ABCA1) and ATP Binding Cassette Subfamily G Member 1 (ABCG1)-mediated cholesterol efflux. Astrocyte-derived ApoE particles interact with Triggering Receptor Expressed on Myeloid Cells 2 (TREM2), Toll Like Receptor 4 (TLr-4), Low-Density-Lipoprotein Receptor (LDLR) and Lipoprotein Lipase (LPL) tethered to Heparan sulfate proteoglycans (HSPG) on the cell surface of microglia. Internalized lipoprotein particles or components provide a source of FFAs, phospholipids and cholesterol to microglia, driving oxidative metabolism and anti-inflammatory functions. FFAs act a ligand for Peroxisome Proliferator Activated Receptors (PPARs), which in turn drives transcription of genes involved in cholesterol efflux (ABCG1 and ABCA1) and lipid uptake (LPL and CD36). Phospholipids are used in the generation of cell membranes, and the FAs can be cleaved by Phospholipase A2 (PLA2) resulting in production of downstream metabolites such as Prostaglandins (PGs). In the absence of lipid uptake low affinity ligands bind TREM2, and glycolysis and pro-inflammatory functions such as increased inflammatory mediator production and neutral lipid accumulation (TG and CE). Cholesterol and myelin breakdown products (24-OHC, 25-OHC, and 27-OHC) from neurons also provide ligands for nuclear receptors (Liver X receptor [LXR] and Retinoic acid receptor [RXR]) that regulate ABCA1, ABCG1, and ApoE transcription and the production of microglial derived Lipoproteins. Created with Biorender.com.

A (SCARA), B (SCARB), or C (SCARC) among others are utilized in both transportation of esterified and non-esterified FAs across the BBB and the monocytic cell membrane. Importantly, these factors are all expressed in microglia, and are regulated during development and disease. CD36, also known as FA translocase (FAT), is a SCARB that plays a major role in microglial LC-FA uptake, oxidized LDL (oxLDL) and fA $\beta$ adhesion as well as activation of the innate immune response and ROS production (Coraci et al., 2002; Doens and Fernández, 2014). Interestingly, CD36 is predominantly expressed in microglia of the cerebellum, which may represent increased requirement for FA substrates in a region of the brain where microglia are relatively sparse and are required to survey larger areas (Grabert et al., 2016). Importantly, FAs are often not transported freely.
They are instead transported in the form of esterified FAs and CE within lipoproteins.

\section{CHOLESTEROL METABOLISM IN MICROGLIA}

Lipoprotein derived $\mathrm{CE}$ is broken down by $\mathrm{CE}$ hydrolase to produce cholesterol and free FAs (FFAs). Although relatively understudied, recent proteomic analysis of specific cell types in the brain has shown that neutral CE hydrolase 1 is abundant in neurons and glia (Viader et al., 2016). Cholesterol is an essential component of the cell membranes that are especially important within the brain. The CNS contains $23 \%$ of the 
body's total cholesterol content; most of which is a major cell membrane component of neurons and myelin (Hamilton et al., 2007). Unlike other organs, most of the cholesterol comes not from the liver, but from synthesis within the brain itself. De novo cholesterol synthesis is not very efficient within neurons, and is primarily achieved by astrocytes (Nieweg et al., 2009). Synthesis of cholesterol, within astrocytes is regulated by sterol regulatory element binding proteins (SREBPs) as well as 3-hydroxy-3-methyl-glutaryl-coenzyme A reductase (HMGCR) (Ye and DeBose-Boyd, 2011). Cholesterol synthesis is vital in the developing CNS, however, in the healthy adult brain, there is a very low level of cholesterol synthesis (Lavrnja et al., 2017). Cholesterol synthesis is high during development due to increased levels of myelinogenesis (Saher et al., 2005), and as previously stated, cholesterol makes up $\sim 28 \%$ of the total lipid volume of myelin (Morell, 1999). In neuroinflammatory diseases, destruction of myelin leads to a buildup of myelin debris that can stimulate microglial phagocytosis, directly inhibit remyelination, and propagates a pro-inflammatory response (Clarner et al., 2012; Kopper and Gensel, 2018). Cholesterol clearance is an important function of microglia (Lavrnja et al., 2017; Cantuti-Castelvetri et al., 2018). In fact, high concentrations of cholesterol are required for the survival of microglia (Bohlen et al., 2017). Additionally, cholesterol is necessary for phagocytosis as well as cytokine release (Churchward and Todd, 2014). Cholesterol homeostasis within the healthy brain also requires its constant elimination from the CNS compartment (Dietschy and Turley, 2004).

The major mechanism for elimination of cholesterol from the brain is its conversion to $24(\mathrm{~S})$-hydroxycholesterol (24-OHC) which freely exits neurons and the brain (Lund et al., 1999). This is achieved by cholesterol 24S-hydroxylase (CYP46A1) which is solely expressed in neurons in healthy brain (Lund et al., 1999). In neuroinflammatory disease states such as $\mathrm{AD}$ and MS, however, CYP46A1 is also highly expressed in both microglia and infiltrating macrophages (Lavrnja et al., 2017). CYP46A1 has been shown to be neuroprotective in the striatum of the R6/2 murine model of Huntington's disease (Boussicault et al., 2016). These findings highlight the importance of cholesterol elimination and its therapeutic potential. What happens when cholesterol elimination is inadequate, and cholesterol accumulates in the microenvironment? Importantly, $\mathrm{CE}$ accumulation is observed in both familial AD (FAD) and sporadic late onset AD (SAD) (van der Kant et al., 2019). Specifically, CE-containing lipids droplets have been shown to accumulate in the SAD brain (Chan et al., 2012). These findings have also been corroborated with observations of increased CE in mouse models of AD (Yang et al., 2014).

In chronic neuroinflammatory states, myelin debris containing phagocytes become "foamy" due to a buildup of FAs, cholesterol, and their breakdown products (CantutiCastelvetri et al., 2018). As previously stated, there is a buildup of $7 \mathrm{KC}$ in the CSF of patients with MS (Diestel et al., 2003; Leoni et al., 2017). 7KC metabolism is not well understood, however studies suggest that $7 \mathrm{KC}$ degradation is facilitated by either 27-hydroxylase or $11 \beta$-hydroxysteroid dehydrogenase type 1 (11ß-HSD1) (Lyons and Brown, 2000; Mitiæ et al., 2013).
Therefore, a buildup of $7 \mathrm{KC}$ is likely associated with ineffective microglial cholesterol metabolism. For example, MS patients have increased CSF cholesterol, likely as a result impaired cholesterol elimination (van de Kraats et al., 2013). These breakdown products are natural ligands for the liver X receptor (LXR) (see Figure 2). When ligand-bound LXR creates a heterodimer with retinoid $\mathrm{X}$ receptor (RXR), which transcriptionally regulates expression of cellular cholesterol efflux proteins such as ApoE and ATP-binding cassette transporters (ABCs) (Zhao and Dahlman-Wright, 2010). ABCA1 and ABCG1 are vital for microglial lipoprotein metabolism as they are responsible for the efflux of cholesterol which gets incorporated into ApoE containing high-density-lipoprotein (HDL)-like particles (Hirsch-Reinshagen et al., 2004). Foamy phagocytes contain increased levels of 24-OHC, 25-OHC, and 27-OHC as well as up-regulated expression of LXR, ABCA1 and ABCG1 (Zhao and Dahlman-Wright, 2010), which may be an attempt to rid foamy microglia or perivascular macrophages of the cholesterol containing myelin debris (Nathanael et al., 2012).

As previously stated, aged microglia have defective phagocytic cholesterol clearance which limits remyelination in MS (CantutiCastelvetri et al., 2018). Myelin debris accumulates in the cells' cytosol as lipid droplets and needle-shaped cholesterol crystals (Cantuti-Castelvetri et al., 2018). Moreover, debris accumulates within microglial lysosomes, leading to lysosomal rupture, and inflammasome stimulation (Cantuti-Castelvetri et al., 2018). Altogether, the balance between cholesterol intake and cholesterol efflux may play a major role in the pathogenesis and progression of neuroinflammatory diseases. Perhaps increased cholesterol efflux may reverse metabolic derangements. For example, a recent study has demonstrated that apolipoprotein A1 (ApoA-1) binding protein (AIBP) binds to TLR4, a component of the innate immune response, within the cell membranes of activated microglia to stimulate removal of excess cholesterol via efflux to HDL or ApoA-1 (see Figure 2) (Woller et al., 2018). Furthermore, intrathecal AIBP reduces TLR4 dimerization and neuroinflammation in the spinal cord in facilitated pain states (Woller et al., 2018). Although microglial cholesterol efflux is important, a major contributor of cholesterol and oxysterol efflux is achieved by astrocytes and neurons (Chen et al., 2013). Astrocytes also use ABCA1 and ABCG1 for cholesterol efflux into lipoproteins, whereas neurons use ABCA1, ABCG1, and ABCG4 (Chen et al., 2013). Cholesterol efflux seems to have therapeutic potential and can be modulated via lipoprotein metabolism.

\section{LIPOPROTEIN METABOLISM IN MICROGLIA}

Tightly regulated lipid metabolism in the CNS is critical for maintaining normal brain functions. However, because lipids such as cholesterol and triglycerides are insoluble in water, they are transported with apoproteins, which creates an emulsion that can be transported to other cells via the extracellular milieu and/or peripheral circulation. The resulting lipoproteins are complex particles composed of a central hydrophobic core of neutral lipids such as CEs and TGs, surrounded 
by an amphipathic monolayer of PLs, free cholesterol, and apolipoproteins.

Although lipoprotein metabolism in the circulation and the CNS vary considerably, it is useful to outline what we have learned from circulating lipoproteins for a clearer understanding of lipoprotein metabolism as a whole. In the peripheral circulation, exogenous lipoprotein metabolism begins with TG-rich chylomicrons (CMs) that are hydrolyzed by LPL to produce $\mathrm{CM}$ remnants, which are then taken up into the liver. The liver initiates endogenous lipoprotein production via the formation of VLDL carrying a range of apoproteins including apolipoprotein B-100 (ApoB-100) and ApoE. VLDL is hydrolyzed further by LPL to form intermediate-densitylipoproteins (IDL) and low-density-lipoproteins (LDL), also carrying ApoB-100 and ApoE, which are then bound to LDL receptors and internalized by cells of key metabolic tissues such as muscle, heart, adipose tissue and liver (Grunfeld and Feingold, 2018). There are no reports of VLDL and LDL in the CNS. However, it is tempting to speculate that lower-density-lipoproteins could penetrate the BBB in pathological conditions such as increased inflammation. In addition, it is possible that larger lipoproteins could have enhanced penetrance in the circumventricular organs of the brain; regions devoid of a typical $\mathrm{BBB}$ with intimate contact between the blood and CSF such as the median eminence (Gross and Weindl, 1987).

In contrast, HDL or HDL-like lipoproteins may traverse the $\mathrm{BBB}$ to enter the CSF under physiologic conditions (Stukas et al., 2014). In the periphery, the first step in HDL formation involves the synthesis of ApoA-I by the liver and intestine. After secretion, ApoA-I acquires cholesterol and PLs that are effluxed by hepatocytes and enterocytes. Cholesterol efflux is regulated by ABCA1, ABCG1 and class $B$ scavenger receptor protein (SR-B1). It is thought that increased intracellular cholesterol leads to oxysterol formation, which triggers the activation of the LXR-RXR heterodimer and $A B C A 1$ and $A B C G 1$ expression in order to efflux/remove cholesterol from the cell. During lipidation, Lecithin-cholesterol acyltransferase (LCAT) transfers a FA from PLs to free cholesterol forming $\mathrm{CE}$, which can then be transferred to the HDLcore (Grunfeld and Feingold, 2018). Sterol O-acyltransferase (ACAT) shares a similar function to LCAT, however it catalyzes the formation of CEs from cholesterol and LC-fatty acyl$\mathrm{CoA}$ and plays a role in hepatic lipoprotein assembly ( $\mathrm{Li}$ et al., 1999). Although ApoA-I is not synthesized in the brain, fluorescently labeled ApoA-I rapidly accumulates in the CSF after intravenous injection (Stukas et al., 2014), highlighting a plausible role in cholesterol delivery to and from the brain (Mahley, 2016).

ApoA-I is a major apoprotein of HDL-like particles in the brain, second only to ApoE. Unlike ApoA-I, ApoE is synthetized within the CNS predominantly by astrocytes (including specialized astrocytic cells, Bergmann glia, tanycytes, pituicytes, and retinal Muller cells) (Boyles et al., 1985). It has been suggested that astrocytes secrete cholesterol-rich ApoE/HDL through an interaction with ABCA1, to provide cholesterol to other cells of the CNS (Ito et al., 2014). Astrocyte derived ApoE/HDL cholesterol is involved in the provision of lipids for axonal growth and regeneration (Vance et al., 2000), and synaptogenesis (Mauch et al., 2001). Importantly, other cell types can increase ApoE production when the need for lipid substrates is greater, such as during development, injury or stress. For example, in mice expressing enhanced GFP (EGFP) ApoE, injured neurons produce high levels of EGFP and ApoE mRNA in the hippocampus (Xu et al., 2006). ApoE is also expressed in type 1 NSCs (Hong et al., 2016), and can directly regulate proliferation and spine density (Hong et al., 2016). Similarly, scRNA-seq studies have shown that microglia express higher levels of ApoE in the embryonic mouse brain (E14.5) (Hammond et al., 2019), during LPC mediated demyelination injury (Hammond et al., 2019), and in the late stages of disease pathogenesis in murine models of AD (Keren-Shaul et al., 2017). Although the precise role of increased ApoE production in microglia is unknown and may be activation-state and/or microglial subpopulation specific, it is likely that increased ApoE may be a response to increased intracellular cholesterol accumulation. For example, during late embryogenesis a significant number of OLs undergo apoptosis (discussed above). In addition to microglial phagocytosis of dying cells and myelin debris, resident microglia may also increase ApoE production concurrently in order to efflux accumulating cholesterols and oxysterols in an attempt to maintain normal microglial function. Although this has not been empirically determined, aging microglia show reduced ApoE production and massive cholesterol accumulation, with major detriment to their phagocytic capacity (Cantuti-Castelvetri et al., 2018). Interestingly, LXR agonists have been shown to reduce neurodegeneration and pathology in neurogenerative animal models, suggesting that ABCA1/ABCG1-mediated cholesterol efflux promotes homeostatic glial functions (Cantuti-Castelvetri et al., 2018).

In addition to cholesterol efflux, apoproteins and lipoproteins have been repeatedly implicated in microglial phagocytosis. For example, ApoE is known to bind to LDL receptors (e.g. LDL receptor [LDLR], LDL Receptor Related Protein 1 [LRP1] and VLDL receptor [VLDLR]) on the surface of microglia and become internalized via receptor-mediated endocytosis (Pocivavsek et al., 2009). Recently, several other cell surface factors that may participate in lipoprotein uptake and/or endocytosis have been identified. TREM2 is a recently discovered $\mathrm{AD}$ risk gene that encodes a single-transmembrane protein that is selectively expressed in the microglia in the CNS (Klesney-Tait et al., 2006). Although the physiological role of TREM2 is still under investigation, it is thought to be protective in the context of $\mathrm{AD}$ since TREM2 deficiency in the 5XFAD mouse model causes increased amyloid deposition (Wang et al., 2015), whereas overexpression of TREM2 in the Amyloid precursor protein/Presenilin-1 (APP)/(PS1) mouse ameliorates neuropathology (Jiang et al., 2014). Since A $\beta$ is known to bind to ApoE, the protection offered by TREM2 maybe conferred through its ability to bind to phospholipids and apoproteins (ApoE and apolipoprotein $\mathrm{J}[\mathrm{ApoJ}]$ ) and to increase phagocytosis (Atagi et al., 2015). TREM2 has been 
shown to interact with $A \beta$-bound lipoproteins to facilitate $A \beta$ uptake by microglia and human macrophages (Yeh et al., 2016). In fact, TREM2 serves as an extracellular lipid and lipoprotein receptor that modulates the inflammatory profile of microglia depending on the affinity of ligand binding (Lessard et al., 2018). Specifically, high avidity ligands, such as lipids and ApoE, bind TREM2 causing phosphorylation of two tyrosine residues within the adjacent transmembrane DNA polymerase III-Activation Protein 12 (DAP12) (Lessard et al., 2018). This initiates a cellular cascade culminating in survival, proliferation, phagocytosis, and motility depending on the ligand (Lessard et al., 2018). Conversely, when TREM2 is associated with low avidity ligands, an intracellular cascade is initiated that may promote inflammatory functions within the cell (Lessard et al., 2018). Recent work suggests that PS and PE from apoptotic neurons (which are not exposed on the surface of healthy cells) act as signals for TREM2-mediated microglial activation (Shirotani et al., 2019). This topic has been more comprehensively reviewed in a number of recent peer-reviewed articles (Jay et al., 2017; Gratuze et al., 2018).

Human ApoE exists as three major isoforms ApoE2, ApoE3 and ApoE4, which are encoded by three allelic variants at a single gene locus on the long arm of chromosome 19. Each isoform differs by 2 amino acid substitutions (ApoE2 [Cys112, Cys158], ApoE3 [Cys112, Arg158], and ApoE4 [Arg112, Arg158]), which significantly alters their lipid and receptor binding affinities (Mahley and Rall, 2000; Wu and Zhao, 2016). ApoE2 has a lower affinity for LDLRs compared to ApoE3 and ApoE4. In addition, ApoE2 and ApoE3 preferentially bind to small, PL-rich HDL whereas ApoE4 preferentially binds to larger, TG-rich lipoproteins (Wu and Zhao, 2016). ApoE3 is present in approximately $75 \%$ of the population and is believed to play a neutral role in $\mathrm{AD}$. In contrast, ApoE2 is relatively rare (5\% incidence) and is considered to be protective against $\mathrm{AD}$ ( $\mathrm{Wu}$ and Zhao, 2016). While the mechanism of this protection is unclear, it is plausible that its differential association with CNS derived HDL-like lipoprotein particles plays a major role. Importantly, the ApoE4 isoform (present in $20 \%$ of the population), is present in nearly $50 \%$ of $\mathrm{AD}$ patients ( $\mathrm{Wu}$ and Zhao, 2016). Although there has been a major research effort attempting to understand the increased risk of $\mathrm{AD}$ conferred by ApoE4, the mechanisms remain elusive. Nonetheless, in murine models of $\mathrm{AD}$, it has been established that ApoE isoforms differentially effect $A \beta$ accumulation in a dose- and isoform- dependent manner, with hippocampal A $\beta$ burden: ApoE2 < ApoE3 < ApoE4 (Castellano et al., 2011). Findings from in vitro studies have shown that ApoE4 can inhibit TREM2 expression in primary murine microglia (Li et al., 2015), therefore potentially blocking the appropriate microglial response to $\mathrm{A} \beta$ accumulation.

ApoE4 is not the only apoprotein associated with increased AD risk. In 2009, two Genome Wide Association Studies (GWAS) identified ApoJ (otherwise known as Clusterin [CLU]) as a novel SAD-risk gene (Harold et al., 2009; Lambert et al., 2009). ApoJ is now considered the third greatest risk factor for SAD.
Although a number of possible mechanisms have been proposed, including its role in TREM2-mediated microglial lipoprotein and $\mathrm{A} \beta$ clearance (Yeh et al., 2016), its precise function is has yet to be determined (Foster et al., 2019).

\section{THE ROLE OF LPL IN MICROGLIA FUNCTION AND METABOLISM}

In the periphery, LPL is primarily involved in the hydrolysis of CM-TG and VLDL-TG. In the murine brain LPL is predominantly expressed in microglia/macrophages and the OPC (Zhang et al., 2014), whereas in the human brain LPL is predominantly expressed in microglia (Zhang et al., 2016). Although the role of LPL in microglia is not well understood, LPL has been repeatedly implicated in $\mathrm{AD}$ pathogenesis in humans. For example, patients with $\mathrm{AD}$ have reduced LPL immunoreactivity in the dentate gyrus and reduced LPL activity in their CSF (Gong et al., 2013). In addition, loss of function LPL polymorphisms have been linked to reduced enzymatic activity, increased VLDL-TG, and increased AD risk (Ren and Ren, 2016). In contrast, patients with a gainof-function LPL polymorphism (447Ter) have increased LPL activity concomitant with lower VLDL-TG, higher HDL, and significantly reduced hippocampal amyloid plaque formation (Baum et al., 2000). Moreover, there is a growing body of literature highlighting the importance of LPL in microglial function. For example, in the 5XFAD mouse model of $\mathrm{AD}$, LPL expression is dramatically increased in DAM (KerenShaul et al., 2017). Importantly, markedly enhanced LPL expression repeatedly observed in microglial subpopulations, particularly in the context of development (Hammond et al., 2019), demyelination (Hammond et al., 2019), and disease (Mathys et al., 2017). In both mouse and human brains, LPL co-localizes with microglia that have internalized $A \beta$ (KerenShaul et al., 2017), suggesting that microglial-LPL may also play a role in $A \beta$ uptake. Indeed, LPL has been shown to directly bind $A \beta$ and play a role in uptake and degradation in astrocytes (Nishitsuji et al., 2011). However, exactly how LPL may interact with $A \beta$ remains to be determined. Since LPL is known to directly interact with LRP1 (Beisiegel et al., 1991; Chappell et al., 1992), which is major receptor for both ApoE and A $\beta$ (Herz and Strickland, 2001), it is plausible that LPL could facilitate $A \beta$ uptake through interaction with other factors expressed on the microglia cell surface. LPL also directly interacts with ApoE (Baum et al., 2000), and it is plausible that the strength of the interaction may vary depending on the isoform's specific lipid binding avidity. The role of LPL in microglia could conceivably link both lipid metabolism and inflammation. We have previously shown that the BV-2 microglia is polarized to a robust inflammatory phenotype following LPL loss, a phenotype that is recapitulated in primary microglia (Bruce et al., 2018). Moreover, loss of LPL shifts the metabolic profile of microglial cell lines toward increased glycolysis and reduced oxidative metabolism, reminiscent of the Warburg-like metabolism observed in inflammatory macrophages (Bruce et al., 2018). Our findings suggest that the importance of LPL in 
microglia may extend beyond phagocytosis of $A \beta$ to regulation of metabolic and inflammatory phenotype.

\section{FUTURE STRATEGIES}

The findings highlighted in this review suggest that lipid and lipoprotein metabolism is a tightly regulated component of microglial immunometabolism. The changes seen in microglial lipid metabolism during damage and disease are some of the most drastic and profound responses that have been observed to date. This suggests that microglial lipid, cholesterol, and lipoprotein metabolism may be novel therapeutic targets for the treatment of CNS disorders such as ND. Since pro-inflammatory microglia and perivascular macrophages that may contribute to ND progression exhibit increased glycolysis but decreased OXPHOS and FAO, it is plausible that modulating substrate utilization in microglia could ameliorate neuroinflammation. Although the optimal method to switch substrate usage in microglia has not been defined, several studies have shown that dietary approaches are sufficient to modulate microglial immunometabolism. Ketogenic diets typically contain very low carbohydrate, but very high fat levels and therefore have the capacity to promote FAO and limit glycolytic flux. In a recent study using a murine model of glaucoma, a ketogenic diet (10.4\% protein, $0.1 \%$ carbohydrate, and $89.5 \%$ fat) was able to reduce markers of microglial activation such as Ionized calcium binding adaptor molecule 1 (Iba1) (HarunOr-Rashid and Inman, 2018). It has also been shown that ketones can directly modulate inflammation in microglia. For example, $\beta$-hydroxybutyrate activates G-protein-coupled receptors 109A (GPR109A) to attenuate NF- $\kappa B$ signaling and pro-inflammatory cytokine production (Fu et al., 2015). These studies suggest that ketogenic diets may be a promising strategy; however, it is important that we do not halt the waves of microglial activation that may be potentially protective in the contexts of certain disease states. In addition, it is critical to carefully evaluate the lipid composition of these

\section{REFERENCES}

Absinta, M., Sati, P., Masuzzo, F., Nair, G., Sethi, V., Kolb, H., et al. (2019). Association of chronic active multiple sclerosis lesions with disability in vivo. 76, 1474-1483. JAMA Neurol. doi: 10.1001/jamaneurol.2019.2399

Ajami, B., Bennett, J. L., Krieger, C., Tetzlaff, W., and Rossi, F. M. (2007). Local self-renewal can sustain CNS microglia maintenance and function throughout adult life. Nat. Neurosci. 10, 1538-1543. doi: 10.1038/nn2014

Aldana, B. I. (2019). Microglia-specific metabolic changes in neurodegeneration. J. Mol. Biol. 431, 1830-1842. doi: 10.1016/j.jmb.2019.03.006

Alliot, F., Godin, I., and Pessac, B. (1999). Microglia derive from progenitors, originating from the yolk sac, and which proliferate in the brain. Dev. Brain Res. 117, 145-152. doi: 10.1016/s0165-3806(99)00113-3

Alliot, F., Lecain, E., Grima, B., and Pessac, B. (1991). Microglial progenitors with a high proliferative potential in the embryonic and adult mouse brain. Proc. Natl. Acad. Sci. U.S.A. 88:1541. doi: 10.1073/pnas.88.4.1541

Allweis, C., Landau, T., Abeles, M., and Magnes, J. (1966). The oxidation of uniformly labelled albumin-bound palmitic acid to $\mathrm{CO} 2$ by the perfused cat brain. J. Neurochem. 13, 795-804. doi: 10.1111/j.1471-4159.1966.tb05874.x diets, since only those including LC-FAs would promote mitochondrial oxidative metabolism. It is also important to note that ketogenic diets may not be optimal for overall metabolic health, and other strategies, such as caloric restriction, and fasting may increase ketone delivery to the brain to downregulate microglial activation without markedly elevating lipid consumption (Loncarevic-Vasiljkovic et al., 2012; Tu et al., 2012; Ghosh et al., 2018).

\section{CONCLUDING REMARKS}

In summary, microglia are dynamic cells that are not only critical for homeostatic brain functions but in mitigating the response to a variety of stimuli such as primary myelination, and demyelination. During their response, microglia tightly regulate lipid and lipoprotein metabolism in order to fuel greater bioenergetics needs, to phagocytose and process lipidrich debris, and to produce precursors for secondary messengers. Although recent studies have galvanized the importance of lipid and lipoprotein metabolism in microglia, this emerging field is likely to reveal further important mechanisms going forward. In addition, further study into the role of lipid metabolism in the polarization of microglial inflammatory status may highlight novel approaches that modulate metabolism to ameliorate neuroinflammatory and NDs.

\section{AUTHOR CONTRIBUTIONS}

$\mathrm{KB}$ devised the framework of the manuscript. BL designed and created the figures. $\mathrm{BL}$ and $\mathrm{KB}$ shared equal parts in drafting and editing the manuscript.

\section{FUNDING}

This work was supported by a CCTSI NCATS KL2 award (KL2TR00253) to KB.

Arterburn, L. M., Hall, E. B., and Oken, H. (2006). Distribution, interconversion, and dose response of n-3 fatty acids in humans. Am. J. Clin. Nutr. 83, 1467s1476s. doi: 10.1093/ajcn/83.6.1467S

Askew, K., Li, K., Olmos-Alonso, A., Garcia-Moreno, F., Liang, Y., Richardson, P., et al. (2017). Coupled proliferation and apoptosis maintain the rapid turnover of microglia in the adult brain. Cell Rep. 18, 391-405. doi: 10.1016/j.celrep.2016. 12.041

Atagi, Y., Liu, C. C., Painter, M. M., Chen, X. F., Verbeeck, C., Zheng, H., et al. (2015). Apolipoprotein $\mathrm{E}$ is a ligand for triggering receptor expressed on myeloid Cells 2 (TREM2). J. Biol. Chem. 290, 26043-26050. doi: 10.1074/jbc. m115.679043

Baik, S. H., Kang, S., Lee, W., Choi, H., Chung, S., Kim, J. I., et al. (2019). A breakdown in metabolic reprogramming causes microglia dysfunction in Alzheimer's disease. Cell Metab. 30, 493-507.e6. doi: 10.1016/j.cmet.2019. 06.005

Barres, B. A., Hart, I. K., Coles, H. S. R., Burne, J. F., Voyvodic, J. T., Richardson, W. D., et al. (1992). Cell death and control of cell survival in the oligodendrocyte lineage. Cell 70, 31-46. doi: 10.1016/0092-8674(92) 90531-g 
Baum, L., Wiebusch, H., and Pang, C. P. (2000). Roles for lipoprotein lipase in Alzheimer's disease: an association study. Microsc. Res. Tech. 50, 291-296. doi: 10.1002/1097-0029(20000815)50:4<291::aid-jemt8>3.0.co;2-1

Bazan, H. E., Bazan, N. G., Feeney-Burns, L., and Berman, E. R. (1990). Lipids in human lipofuscin-enriched subcellular fractions of two age populations. Comparison with rod outer segments and neural retina. Invest. Ophthalmol. Vis. Sci. 31, 1433-1443.

Beckers, L., Geric, I., Stroobants, S., Beel, S., Van Damme, P., D’Hooge, R., et al. (2019). Microglia lacking a peroxisomal beta-oxidation enzyme chronically alter their inflammatory profile without evoking neuronal and behavioral deficits. J. Neuroinflammation 16:61. doi: 10.1186/s12974-019-1442-3

Beisiegel, U., Weber, W., and Bengtsson-Olivecrona, G. (1991). Lipoprotein lipase enhances the binding of chylomicrons to low density lipoprotein receptorrelated protein. Proc. Natl. Acad. Sci. U.S.A. 88, 8342-8346. doi: 10.1073/pnas. 88.19.8342

Bélanger, M., Allaman, I., and Magistretti, P. J. (2011). Brain energy metabolism: focus on astrocyte-neuron metabolic cooperation. Cell Metab. 14, 724-738. doi: 10.1016/j.cmet.2011.08.016

Bennett, M. L., and Bennett, F. C. (2020). The influence of environment and origin on brain resident macrophages and implications for therapy. Nat. Neurosci. 23, 157-166. doi: 10.1038/s41593-019-0545-6

Bernardo, A., Ajmone-Cat, M. A., Levi, G., and Minghetti, L. (2003). 15-deoxydelta12,14-prostaglandin J2 regulates the functional state and the survival of microglial cells through multiple molecular mechanisms. J. Neurochem. 87, 742-751. doi: 10.1046/j.1471-4159.2003.02045.x

Bernardo, A., Levi, G., and Minghetti, L. (2000). Role of the peroxisome proliferator-activated receptor-gamma (PPAR-gamma) and its natural ligand 15-deoxy-Delta12, 14-prostaglandin $\mathrm{J} 2$ in the regulation of microglial functions. Eur. J. Neurosci. 12, 2215-2223. doi: 10.1046/j.1460-9568.2000.00110.x

Berson, E. L., Rosner, B., Sandberg, M. A., Weigel-DiFranco, C., and Willett, W. C. (2012). $\omega-3$ intake and visual acuity in patients with retinitis pigmentosa receiving vitamin A. Arch. Ophthalmol. 130, 707-711.

Bhattacharyya, R., and Kovacs, D. M. (2010). ACAT inhibition and amyloid beta reduction. Biochim. Biophys. Acta 1801, 960-965. doi: 10.1016/j.bbalip.2010. 04.003

Bialas, A. R., and Stevens, B. (2013). TGF-beta signaling. Nat. Neurosci. 16, 1773-1782. doi: 10.1038/nn.3560

Bilimoria, P. M., and Stevens, B. (2015). Microglia function during brain development: new insights from animal models. Brain Res. 1617, 7-17. doi: 10.1016/j.brainres.2014.11.032

Bjornevik, K., Chitnis, T., Ascherio, A., and Munger, K. L. (2017). Polyunsaturated fatty acids and the risk of multiple sclerosis. Mult. Scler. 23, 1830-1838. doi: $10.1177 / 1352458517691150$

Bohlen, C. J., Bennett, F. C., Tucker, A. F., Collins, H. Y., Mulinyawe, S. B., and Barres, B. A. (2017). Diverse requirements for microglial survival, specification, and function revealed by defined-medium cultures. Neuron 94, 759-773.e8. doi: 10.1016/j.neuron.2017.04.043

Boussicault, L., Alves, S., Lamazière, A., Planques, A., Heck, N., Moumné, L., et al. (2016). CYP46A1, the rate-limiting enzyme for cholesterol degradation, is neuroprotective in Huntington's disease. Brain J. Neurol. 139, 953-970. doi: 10.1093/brain/awv384

Boyles, J. K., Pitas, R. E., Wilson, E., Mahley, R. W., and Taylor, J. M. (1985). Apolipoprotein E associated with astrocytic glia of the central nervous system and with nonmyelinating glia of the peripheral nervous system. J. Clin. Invest. 76, 1501-1513. doi: 10.1172/jci112130

Brigham, E. P., Woo, H., McCormack, M., Rice, J., Koehler, K., Vulcain, T., et al. (2019). Omega-3 and Omega-6 intake modifies asthma severity and response to indoor air pollution in children. Am. J. Respir. Crit. Care Med. 199, 1478-1486. doi: 10.1164/rccm.201808-1474OC

Bruce, K. D., Gorkhali, S., Given, K., Coates, A. M., Boyle, K. E., Macklin, W. B., et al. (2018). Lipoprotein lipase is a feature of alternatively-activated microglia and may facilitate lipid uptake in the CNS during demyelination. Front. Mol. Neurosci. 11:57. doi: 10.3389/fnmol.2018.00057

Bruce, K. D., Zsombok, A., and Eckel, R. H. (2017). Lipid processing in the brain: a key regulator of systemic metabolism. Front. Endocrinol. (Lausanne) 8:60. doi: $10.3389 /$ fendo. 2017.00060

Bryleva, E. Y., Rogers, M. A., Chang, C. C., Buen, F., Harris, B. T., Rousselet, E., et al. (2010). ACAT1 gene ablation increases 24(S)-hydroxycholesterol content in the brain and ameliorates amyloid pathology in mice with AD. Proc. Natl. Acad. Sci. U.S.A. 107, 3081-3086. doi: 10.1073/pnas.0913828107

Butovsky, O., Jedrychowski, M. P., Moore, C. S., Cialic, R., Lanser, A. J., Gabriely, G., et al. (2014). Identification of a unique TGF-beta-dependent molecular and functional signature in microglia. Nat. Neurosci. 17, 131-143. doi: 10.1038/nn. 3599

Button, E. B., Mitchell, A. S., Domingos, M. M., Chung, J. H., Bradley, R. M., Hashemi, A., et al. (2014). Microglial cell activation increases saturated and decreases monounsaturated fatty acid content, but both lipid species are proinflammatory. Lipids 49, 305-316. doi: 10.1007/s11745-014-3882-y

Calder, P. C. (2015). Marine omega-3 fatty acids and inflammatory processes: effects, mechanisms and clinical relevance. Biochim. Biophys. Acta 1851, 469484. doi: 10.1016/j.bbalip.2014.08.010

Cannon, T. D., Chung, Y., He, G., Sun, D., Jacobson, A., van Erp, T. G., et al. (2015). Progressive reduction in cortical thickness as psychosis develops: a multisite longitudinal neuroimaging study of youth at elevated clinical risk. Biol. Psychiatry 77, 147-157. doi: 10.1016/j.biopsych.2014.05.023

Cantuti-Castelvetri, L., Fitzner, D., Bosch-Queralt, M., Weil, M.-T., Su, M., and Sen, P. (2018). Defective cholesterol clearance limits remyelination in the aged central nervous system. Science 359:684. doi: 10.1126/science. aan 4183

Cardona, A. E., Pioro, E. P., Sasse, M. E., Kostenko, V., Cardona, S. M., Dijkstra, I. M., et al. (2006). Control of microglial neurotoxicity by the fractalkine receptor. Nat. Neurosci. 9, 917-924. doi: 10.1038/nn1715

Carroll, M. C. (2004). The complement system in B cell regulation. Mol. Immunol. 41, 141-146. doi: 10.1016/j.molimm.2004.03.017

Castellano, J. M., Kim, J., Stewart, F. R., Jiang, H., DeMattos, R. B., Patterson, B. W., et al. (2011). Human apoE isoforms differentially regulate brain amyloidbeta peptide clearance. Sci. Transl. Med. 3:89ra57. doi: 10.1126/scitranslmed. 3002156

Chan, J. P., Wong, B. H., Chin, C. F., Galam, D. L. A., Foo, J. C., Wong, L. C., et al. (2018). The lysolipid transporter Mfsd2a regulates lipogenesis in the developing brain. PLoS Biol. 16:e2006443. doi: 10.1371/journal.pbio.2006443

Chan, R. B., Oliveira, T. G., Cortes, E. P., Honig, L. S., Duff, K. E., Small, S. A., et al. (2012). Comparative lipidomic analysis of mouse and human brain with Alzheimer disease. J. Biol. Chem. 287, 2678-2688. doi: 10.1074/jbc.M111. 274142

Chappell, D. A., Fry, G. L., Waknitz, M. A., Iverius, P. H., Williams, S. E., and Strickland, D. K. (1992). The low density lipoprotein receptor-related protein/alpha 2-macroglobulin receptor binds and mediates catabolism of bovine milk lipoprotein lipase. J. Biol. Chem. 267, 25764-25767.

Chausse, B., Kakimoto, P. A., Caldeira-da-Silva, C. C., Chaves-Filho, A. B., Yoshinaga, M. Y., da Silva, R. P., et al. (2019). Distinct metabolic patterns during microglial remodeling by oleate and palmitate. Biosci. Rep. 39:BSR20190072. doi: 10.1042/BSR20190072

Chen, C. T., and Bazinet, R. P. (2015). $\beta$-oxidation and rapid metabolism, but not uptake regulate brain eicosapentaenoic acid levels. Prostaglandins Leukot. Essent. Fatty Acids 92, 33-40. doi: 10.1016/j.plefa.2014.05.007

Chen, J., Zhang, X., Kusumo, H., Costa, L. G., and Guizzetti, M. (2013). Cholesterol efflux is differentially regulated in neurons and astrocytes: implications for brain cholesterol homeostasis. Biochim. Biophys. Acta 1831, 263-275. doi: 10.1016/j. bbalip.2012.09.007

Chen, S., Zhang, H., Pu, H., Wang, G., Li, W., Leak, R. K., et al. (2014). n-3 PUFA supplementation benefits microglial responses to myelin pathology. Sci. Rep. 4:7458. doi: 10.1038/srep07458

Chen, X., Chen, C., Fan, S., Wu, S., Yang, F., Fang, Z., et al. (2018). Omega-3 polyunsaturated fatty acid attenuates the inflammatory response by modulating microglia polarization through SIRT1-mediated deacetylation of the HMGB1/NF-kappaB pathway following experimental traumatic brain injury. J. Neuroinflammation 15:116. doi: 10.1186/s12974-018-1151-3

Chu, Y., Jin, X., Parada, I., Pesic, A., Stevens, B., Barres, B., et al. (2010). Enhanced synaptic connectivity and epilepsy in C1q knockout mice. Proc. Natl. Acad. Sci. U.S.A. 107, 7975-7980. doi: 10.1073/pnas.0913449107

Churchward, M. A., and Todd, K. G. (2014). Statin treatment affects cytokine release and phagocytic activity in primary cultured microglia through two separable mechanisms. Mol. Brain 7:85. doi: 10.1186/s13041-014-0085-7

Clarner, T., Diederichs, F., Berger, K., Denecke, B., Gan, L., van der Valk, P., et al. (2012). Myelin debris regulates inflammatory responses in an experimental 
demyelination animal model and multiple sclerosis lesions. Glia 60, 1468-1480. doi: $10.1002 /$ glia.22367

Colonna, M., and Butovsky, O. (2017). Microglia function in the central nervous System during health and neurodegeneration. Annu. Rev. Immunol. 35, 441468. doi: 10.1146/annurev-immunol-051116-052358

Coraci, I. S., Husemann, J., Berman, J. W., Hulette, C., Dufour, J. H., Campanella, G. K., et al. (2002). CD36, a class B scavenger receptor, is expressed on microglia in Alzheimer's disease brains and can mediate production of reactive oxygen species in response to beta-amyloid fibrils. Am. J. Pathol. 160, 101-112. doi: 10.1016/s0002-9440(10)64354-4

Crotti, A., and Ransohoff, R. M. (2016). Microglial physiology and pathophysiology: insights from genome-wide transcriptional profiling. Immunity 44, 505-515. doi: 10.1016/j.immuni.2016.02.013

Croze, E., Yamaguchi, K. D., Knappertz, V., Reder, A. T., and Salamon, H. (2013). Interferon-beta-1b-induced short- and long-term signatures of treatment activity in multiple sclerosis. Pharmacogenomics J. 13, 443-451. doi: 10.1038/ tpj.2012.27

Davalos, D., Grutzendler, J., Yang, G., Kim, J. V., Zuo, Y., Jung, S., et al. (2005). ATP mediates. Nat. Neurosci. 8, 752-758.

De, S., Van Deren, D., Peden, E., Hockin, M., Boulet, A., Titen, S., et al. (2018). Two distinct ontogenies confer heterogeneity to mouse brain microglia. Development 145:dev152306. doi: 10.1242/dev.152306

Dhopeshwarkar, G. A., and Mead, J. F. (1970). Fatty acid uptake by the brain. 3 . Incorporation of (1-14C)oleic acid into the adult rat brain. Biochim. Biophys. Acta 210, 250-256.

Dhopeshwarkar, G. A., Subramanian, C., and Mead, J. F. (1971). Fatty acid uptake by the brain. IV. Incorporation of (I-14C)linoleic acid into the adult rat brain. Biochim. Biophys. Acta 231, 8-14. doi: 10.1016/0005-2760(71) 90250-5

Di Paolo, G., and Kim, T.-W. (2011). Linking lipids to Alzheimer's disease: cholesterol and beyond. Nat. Rev. Neurosci. 12, 284-296. doi: 10.1038/nrn3012

Diestel, A., Aktas, O., Hackel, D., Hake, I., Meier, S., Raine, C. S., et al. (2003). Activation of microglial poly(ADP-ribose)-polymerase-1 by cholesterol breakdown products during neuroinflammation: a link between demyelination and neuronal damage. J. Exp. Med. 198, 1729-1740. doi: 10.1084/jem.20030975

Dietschy, J. M., and Turley, S. D. (2004). Thematic review series: brain Lipids. Cholesterol metabolism in the central nervous system during early development and in the mature animal. J. Lipid Res. 45, 1375-1397. doi: 10.1194/jlr.r400004-jlr200

Dimas, P., Montani, L., Pereira, J. A., Moreno, D., Trötzmüller, M., Gerber, J., et al. (2019). CNS myelination and remyelination depend on fatty acid synthesis by oligodendrocytes. eLife 8:e44702. doi: 10.7554/eLife.44702

Doens, D., and Fernández, P. L. (2014). Microglia receptors and their implications in the response to amyloid $\beta$ for Alzheimer's disease pathogenesis. J. Neuroinflammation 11:48. doi: 10.1186/1742-2094-11-48

Ebert, D., Haller, R. G., and Walton, M. E. (2003). Energy contribution of octanoate to intact rat brain metabolism measured by $13 \mathrm{C}$ nuclear magnetic resonance spectroscopy. J. Neurosci. 23, 5928-5935. doi: 10.1523/jneurosci.23-13-05928. 2003

Ehehalt, R., Keller, P., Haass, C., Thiele, C., and Simons, K. (2003). Amyloidogenic processing of the Alzheimer beta-amyloid precursor protein depends on lipid rafts. J. Cell Biol. 160, 113-123. doi: 10.1083/jcb.200207113

Esposito, G., Giovacchini, G., Liow, J. S., Bhattacharjee, A. K., Greenstein, D., Schapiro, M., et al. (2008). Imaging neuroinflammation in Alzheimer's disease with radiolabeled arachidonic acid and PET. J. Nucl. Med. 49, 1414-1421. doi: 10.2967/jnumed.107.049619

Fadok, V. A., Bratton, D. L., Rose, D. M., Pearson, A., Ezekewitz, R. A., and Henson, P. M. (2000). A receptor for phosphatidylserine-specific clearance of apoptotic cells. Nature 405, 85-90. doi: 10.1038/35011084

Feingold, K. R., Shigenaga, J. K., Kazemi, M. R., McDonald, C. M., Patzek, S. M., Cross, A. S., et al. (2012). Mechanisms of triglyceride accumulation in activated macrophages. J. Leukoc. Biol. 92, 829-839. doi: 10.1189/jlb.1111537

Foley, P. (2010). Lipids in Alzheimer's disease: a century-old story. Biochim. Biophys. Acta 1801, 750-753. doi: 10.1016/j.bbalip.2010.05.004

Forman, B. M., Tontonoz, P., Chen, J., Brun, R. P., Spiegelman, B. M., and Evans, R. M. (1995). 15-Deoxy-delta 12, 14-prostaglandin J2 is a ligand for the adipocyte determination factor PPAR gamma. Cell 83, 803-812. doi: 10.1016/ 0092-8674(95)90193-0
Foster, E. M., Dangla-Valls, A., Lovestone, S., Ribe, E. M., and Buckley, N. J. (2019). Clusterin in Alzheimer's disease: mechanisms, genetics, and lessons from other pathologies. Front. Neurosci. 13:164. doi: 10.3389/fnins.2019.00164

Fricker, M., Neher, J. J., Zhao, J. W., Thery, C., Tolkovsky, A. M., and Brown, G. C. (2012). MFG-E8 mediates primary phagocytosis of viable neurons during neuroinflammation. J. Neurosci. 32, 2657-2666. doi: 10.1523/JNEUROSCI. 4837-11.2012

Fu, R., Shen, Q., Xu, P., Luo, J. J., and Tang, Y. (2014). Phagocytosis of microglia in the central nervous system diseases. Mol. Neurobiol. 49, 1422-1434. doi: 10.1007/s12035-013-8620-6

Fu, S. P., Wang, J. F., Xue, W. J., Liu, H. M., Liu, B. R., Zeng, Y. L., et al. (2015). Anti-inflammatory effects of BHBA in both in vivo and in vitro Parkinson's disease models are mediated by GPR109A-dependent mechanisms. J. Neuroinflammation 12:9. doi: 10.1186/s12974-014-0230-3

Fumagalli, M., Lombardi, M., Gressens, P., and Verderio, C. (2018). How to reprogram microglia toward beneficial functions. Glia 66, 2531-2549. doi: 10. 1002/glia.23484

Gasque, P. (2004). Complement: a unique innate immune sensor for danger signals. Mol. Immunol. 41, 1089-1098. doi: 10.1016/j.molimm.2004.06.011

Ghosh, S., Castillo, E., Frias, E. S., and Swanson, R. A. (2018). Bioenergetic regulation of microglia. Glia 66, 1200-1212. doi: 10.1002/glia.23271

Gill, E. L., Raman, S., Yost, R. A., Garrett, T. J., and Vedam-Mai, V. (2018). 1-Carnitine inhibits lipopolysaccharide-induced nitric oxide production of SIM-A9 microglia cells. ACS Chem. Neurosci. 9, 901-905. doi: 10.1021/ acschemneuro.7b00468

Ginhoux, F., Greter, M., Leboeuf, M., Nandi, S., See, P., Gokhan, S., et al. (2010). Fate mapping analysis reveals that adult microglia derive from primitive macrophages. Science 330, 841-845. doi: 10.1126/science.1194637

Glantz, L. A., and Lewis, D. A. (2000). Decreased dendritic spine density on prefrontal cortical pyramidal neurons in schizophrenia. Arch. Gen. Psychiatry 57, 65-73.

Glausier, J. R., and Lewis, D. A. (2013). Dendritic spine pathology in schizophrenia. Neuroscience 251, 90-107. doi: 10.1016/j.neuroscience.2012.04.044

Goldmann, T., Wieghofer, P., Jordão, M. J. C., Prutek, F., Hagemeyer, N., Frenzel, K., et al. (2016). Origin, fate and dynamics of macrophages at central nervous system interfaces. Nat. Immunol. 17:797. doi: 10.1038/ni.3423

Gong, H., Dong, W., Rostad, S. W., Marcovina, S. M., Albers, J. J., Brunzell, J. D., et al. (2013). Lipoprotein lipase (LPL) is associated with neurite pathology and its levels are markedly reduced in the dentate gyrus of Alzheimer's disease brains. J. Histochem. Cytochem. 61, 857-868. doi: 10.1369/0022155413505601

Grabert, K., Michoel, T., Karavolos, M. H., Clohisey, S., Baillie, J. K., Stevens, M. P., et al. (2016). Microglial brain region-dependent diversity and selective regional sensitivities to aging. Nat. Neurosci. 19, 504-516. doi: 10.1038/nn.4222

Gratuze, M., Leyns, C. E. G., and Holtzman, D. M. (2018). New insights into the role of TREM2 in Alzheimer's disease. Mol. Neurodegener. 13:66. doi: 10.1186/ s13024-018-0298-9

Green, D. R., Oguin, T. H., and Martinez, J. (2016). The clearance of dying cells: table for two. Cell Death Differ. 23, 915-926. doi: 10.1038/cdd.2015.172

Gross, P. M., and Weindl, A. (1987). Peering through the windows of the brain. J. Cereb. Blood Flow Metab. 7, 663-672. doi: 10.1038/jcbfm.1987.120

Grunfeld, C., and Feingold, K. R. (2018). Introduction to Lipids and Lipoproteins. [Updated 2018 Feb 2]. South Dartmouth, MA: MDText.com, Inc.

Haage, V., Semtner, M., Vidal, R. O., Hernandez, D. P., Pong, W. W., Chen, Z., et al. (2019). Comprehensive gene expression meta-analysis identifies signature genes that distinguish microglia from peripheral monocytes/macrophages in health and glioma. Acta Neuropathol. Commun. 7:20. doi: 10.1186/s40478-019$0665-y$

Hamilton, J. A., Hillard, C. J., Spector, A. A., and Watkins, P. A. (2007). Brain uptake and utilization of fatty acids, lipids and lipoproteins: application to neurological disorders. J. Mol. Neurosci. 33, 2-11. doi: 10.1007/s12031-0070060-1

Hammond, T. R., Dufort, C., Dissing-Olesen, L., Giera, S., Young, A., Wysoker, A., et al. (2019). Single-cell RNA sequencing of microglia throughout the mouse lifespan and in the injured brain reveals complex cell-state changes. Immunity 50, 253-271.e6. doi: 10.1016/j.immuni.2018.11.004

Harold, D., Abraham, R., Hollingworth, P., Sims, R., Gerrish, A., Hamshere, M. L., et al. (2009). Genome-wide association study identifies variants at CLU and PICALM associated with Alzheimer's disease. Nat. Genet. 41, 1088-1093. 
Harrison, J. K., Jiang, Y., Chen, S., Xia, Y., Maciejewski, D., McNamara, R. K., et al. (1998). Role for neuronally derived fractalkine in mediating interactions between neurons and CX3CR1-expressing microglia. Proc. Natl. Acad. Sci. U.S.A. 95, 10896-10901. doi: 10.1073/pnas.95.18.10896

Harun-Or-Rashid, M., and Inman, D. M. (2018). Reduced AMPK activation and increased HCAR activation drive anti-inflammatory response and neuroprotection in glaucoma. J. Neuroinflammation 15:313. doi: 10.1186/ s12974-018-1346-7

Hattori, C., Asai, M., Onishi, H., Sasagawa, N., Hashimoto, Y., Saido, T. C., et al. (2006). BACE1 interacts with lipid raft proteins. J. Neurosci. Res. 84, 912-917. doi: 10.1002/jnr.20981

Hennig, B., and Watkins, B. A. (1989). Linoleic acid and linolenic acid: effect on permeability properties of cultured endothelial cell monolayers. Am. J. Clin. Nutr. 49, 301-305. doi: 10.1093/ajcn/49.2.301

Herz, J., and Strickland, D. K. (2001). LRP: a multifunctional. J. Clin. Invest. 108, 779-784. doi: 10.1172/jci200113992

Hirsch-Reinshagen, V., Zhou, S., Burgess, B. L., Bernier, L., McIsaac, S. A., Chan, J. Y., et al. (2004). Deficiency of ABCA1 impairs apolipoprotein E metabolism in brain. J. Biol. Chem. 279, 41197-41207. doi: 10.1074/jbc.m407962200

Hong, S., Washington, P. M., Kim, A., Yang, C. P., Yu, T. S., and Kernie, S. G. (2016). Apolipoprotein E regulates injury-induced activation of hippocampal neural stem and progenitor cells. J. Neurotrauma 33, 362-374. doi: 10.1089/ neu.2014.3860

Hopperton, K. E., Trepanier, M. O., Giuliano, V., and Bazinet, R. P. (2016). Brain omega-3 polyunsaturated fatty acids modulate microglia cell number and morphology in response to intracerebroventricular amyloid-beta 1-40 in mice. J. Neuroinflammation 13:257.

Ito, J., Nagayasu, Y., Miura, Y., Yokoyama, S., and Michikawa, M. (2014). Astrocytes endogenous apoE generates HDL-like lipoproteins using previously synthesized cholesterol through interaction with ABCA1. Brain Res. 1570, 1-12. doi: 10.1016/j.brainres.2014.04.037

Jakovcevski, I., Filipovic, R., Mo, Z., Rakic, S., and Zecevic, N. (2009). Oligodendrocyte development and the onset of myelination in the human fetal brain. Front. Neuroanat. 3:5. doi: 10.3389/neuro.05.005.2009

Janda, E., Boi, L., and Carta, A. R. (2018). Microglial phagocytosis and its regulation: a therapeutic target in Parkinson's disease? Front. Mol. Neurosci. 11:144. doi: 10.3389/fnmol.2018.00144

Jay, T. R., von Saucken, V. E., and Landreth, G. E. (2017). TREM2 in neurodegenerative diseases. Mol. Neurodegener. 12:56. doi: 10.1186/s13024017-0197-5

Jenkins, C. M., Mancuso, D. J., Yan, W., Sims, H. F., Gibson, B., and Gross, R. W. (2004). Identification, cloning, expression, and purification of three novel human calcium-independent phospholipase A2 family members possessing triacylglycerol lipase and acylglycerol transacylase activities. J. Biol. Chem. 279, 48968-48975. doi: 10.1074/jbc.m407841200

Jha, A. K., Huang, S. C., Sergushichev, A., Lampropoulou, V., Ivanova, Y., Loginicheva, E., et al. (2015). Network integration of parallel metabolic and transcriptional data reveals metabolic modules that regulate macrophage polarization. Immunity 42, 419-430. doi: 10.1016/j.immuni.2015.02.005

Jiang, T., Tan, L., Zhu, X. C., Zhang, Q. Q., Cao, L., Tan, M. S., et al. (2014). Upregulation of TREM2 ameliorates neuropathology and rescues spatial cognitive impairment in a transgenic mouse model of Alzheimer's disease. Neuropsychopharmacology 39, 2949-2962. doi: 10.1038/npp.2014.164

Jung, S., Aliberti, J., Graemmel, P., Sunshine, M. J., Kreutzberg, G. W., Sher, A., et al. (2000). Analysis of fractalkine receptor CX(3)CR1 function by targeted deletion and green fluorescent protein reporter gene insertion. Mol. Cell. Biol. 20, 4106-4114. doi: 10.1128/mcb.20.11.4106-4114.2000

Kang, E. B., Koo, J. H., Jang, Y. C., Yang, C. H., Lee, Y., Cosio-Lima, L. M., et al. (2016). Neuroprotective effects of endurance exercise against high-fat dietinduced hippocampal neuroinflammation. J. Neuroendocrinol. 28. doi: 10.1111/ jne. 12385

Kang, J. X. (2007). Fat-1 transgenic mice: a new model for omega-3 research. Prostaglandins Leukot. Essent. Fatty Acids 77, 263-267. doi: 10.1016/j.plefa. 2007.10.010

Kauppinen, T. M., and Swanson, R. A. (2005). Poly(ADP-Ribose) polymerase-1 promotes microglial activation, proliferation, and matrix metalloproteinase-9mediated neuron death. J. Immunol. 174:2288. doi: 10.4049/jimmunol.174.4. 2288
Kelly, B., and O’Neill, L. A. (2015). Metabolic reprogramming in macrophages and dendritic cells in innate immunity. Cell Res. 25, 771-784. doi: 10.1038/cr. 2015.68

Keren-Shaul, H., Spinrad, A., Weiner, A., Matcovitch-Natan, O., Dvir-Szternfeld, R., Ulland, T. K., et al. (2017). A unique microglia type associated with restricting development of Alzheimer's disease. Cell 169, 1276-1290.e17. doi: 10.1016/j.cell.2017.05.018

Kierdorf, K., Erny, D., Goldmann, T., Sander, V., Schulz, C., Perdiguero, E. G., et al. (2013). Microglia emerge from erythromyeloid precursors via Pu.1- and Irf8-dependent pathways. Nat. Neurosci. 16, 273-280. doi: 10.1038/nn.3318

Klesney-Tait, J., Turnbull, I. R., and Colonna, M. (2006). The TREM receptor family and signal integration. Nat. Immunol. 7, 1266-1273. doi: 10.1038/nil411

Kliewer, S. A., Lenhard, J. M., Willson, T. M., Patel, I., Morris, D. C., and Lehmann, J. M. (1995). A prostaglandin J2 metabolite binds peroxisome proliferatoractivated receptor gamma and promotes adipocyte differentiation. Cell 83, 813-819. doi: 10.1016/0092-8674(95)90194-9

Kopper, T. J., and Gensel, J. C. (2018). Myelin as an inflammatory mediator: myelin interactions with complement, macrophages, and microglia in spinal cord injury. J. Neurosci. Res. 96, 969-977. doi: 10.1002/jnr.24114

Kumar, M., Roe, K., Nerurkar, P. V., Orillo, B., Thompson, K. S., Verma, S., et al. (2014). Reduced immune cell infiltration and increased pro-inflammatory mediators in the brain of Type 2 diabetic mouse model infected with West Nile virus. J. Neuroinflammation 11:80. doi: 10.1186/1742-2094-11-80

Lacor, P. N., Buniel, M. C., Chang, L., Fernandez, S. J., Gong, Y., Viola, K. L., et al. (2004). Synaptic Targeting by Alzheimer's-Related Amyloid $\beta$ Oligomers. J. Neurosci. 24, 10191-10200. doi: 10.1523/jneurosci.3432-04.2004

LaDu, M. J., Falduto, M. T., Manelli, A. M., Reardon, C. A., Getz, G. S., and Frail, D. E. (1994). Isoform-specific binding of apolipoprotein E to beta-amyloid. J. Biol. Chem. 269, 23403-23406.

Lambert, J. C., Heath, S., Even, G., Campion, D., Sleegers, K., Hiltunen, M., et al. (2009). Genome-wide association study identifies variants at CLU and CR1 associated with Alzheimer's disease. Nat. Genet. 41, 1094-1099. doi: 10.1038/ ng.439

Langston, P. K., Shibata, M., and Horng, T. (2017). Metabolism supports macrophage activation. Front. Immunol. 8:61. doi: 10.3389/fimmu.2017.00061

Lavrnja, I., Smiljanic, K., Savic, D., Mladenovic-Djordjevic, A., Tesovic, K., Kanazir, S., et al. (2017). Expression profiles of cholesterol metabolism-related genes are altered during development of experimental autoimmune encephalomyelitis in the rat spinal cord. Sci. Rep. 7:2702. doi: 10.1038/s41598-017-02638-8

Lawson, L. J., Perry, V. H., Dri, P., and Gordon, S. (1990). Heterogeneity in the distribution and morphology of microglia in the normal adult mouse brain. Neuroscience 39, 151-170. doi: 10.1016/0306-4522(90)90229-w

Layé, S., Madore, C., St-Amour, I., Delpech, J., Joffre, C., Nadjar, A., et al. (2015). N3 polyunsaturated fatty acid and neuroinflammation in aging and Alzheimer's disease. Nutr. Aging 3, 33-47. doi: 10.3233/nua- 150049

Layé, S., Nadjar, A., Joffre, C., and Bazinet, R. P. (2018). Anti-inflammatory effects of omega-3 fatty acids in the brain: physiological mechanisms and relevance to pharmacology. Pharmacol. Rev. 70:12. doi: 10.1124/pr.117.014092

Leoni, V., Nury, T., Vejux, A., Zarrouk, A., Caccia, C., Debbabi, M., et al. (2017). Mitochondrial dysfunctions in 7-ketocholesterol-treated $158 \mathrm{~N}$ oligodendrocytes without or with alpha-tocopherol: impacts on the cellular profil of tricarboxylic cycle-associated organic acids, long chain saturated and unsaturated fatty acids, oxysterols, cholesterol and cholesterol precursors. J. Steroid Biochem. Mol. Biol. 169, 96-110. doi: 10.1016/j.jsbmb.2016.03.029

Lessard, C. B., Malnik, S. L., Zhou, Y., Ladd, T. B., Cruz, P. E., Ran, Y., et al. (2018). High-affinity interactions and signal transduction between Abeta oligomers and TREM2. EMBO Mol. Med. 10:e9027. doi: 10.15252/emmm.201809027

Li, B. L., Li, X. L., Duan, Z. J., Lee, O., Lin, S., Ma, Z. M., et al. (1999). Human acyl-CoA:cholesterol acyltransferase-1 (ACAT-1) gene organization and evidence that the 4.3-kilobase ACAT-1 mRNA is produced from two different chromosomes. J. Biol. Chem. 274, 11060-11071. doi: 10.1074/jbc.274. 16.11060

Li, Q., and Barres, B. A. (2018). Microglia and macrophages in brain homeostasis and disease. Nat. Rev. Immunol. 18, 225-242. doi: 10.1038/nri.2017.125

Li, Q., Cheng, Z., Zhou, L., Darmanis, S., Neff, N. F., Okamoto, J., et al. (2019). Developmental heterogeneity of microglia and brain myeloid cells revealed by deep single-cell RNA sequencing. Neuron 101, 207-223.e10. doi: 10.1016/j. neuron.2018.12.006 
Li, X., Montine, K. S., Keene, C. D., and Montine, T. J. (2015). Different mechanisms of apolipoprotein $\mathrm{E}$ isoform-dependent modulation of prostaglandin E2 production and triggering receptor expressed on myeloid cells 2 (TREM2) expression after innate immune activation of microglia. FASEB J. 29, 1754-1762. doi: 10.1096/fj.14-262683

Li, Z., Zhang, J., and Sun, H. (2015). Increased plasma levels of phospholipid in Parkinson's disease with mild cognitive impairment. J. Clin. Neurosci. 22, 1268-1271. doi: 10.1016/j.jocn.2015.02.013

Liang, K. J., Lee, J. E., Wang, Y. D., Ma, W., Fontainhas, A. M., Fariss, R. N., et al. (2009). Regulation of dynamic behavior of retinal microglia by CX3CR1 signaling. Invest. Ophthalmol. Vis. Sci. 50, 4444-4451. doi: 10.1167/iovs.083357

Litman, G. W., Cannon, J. P., and Rast, J. P. (2005). New insights into alternative mechanisms of immune receptor diversification. Adv. Immunol. 87, 209-236. doi: 10.1016/s0065-2776(05)87006-3

Loncarevic-Vasiljkovic, N., Pesic, V., Todorovic, S., Popic, J., Smiljanic, K., Milanovic, D., et al. (2012). Caloric restriction suppresses microglial activation and prevents neuroapoptosis following cortical injury in rats. PLoS One 7:e37215. doi: 10.1371/journal.pone.0037215

Lukiw, W. J., and Bazan, N. G. (2000). Neuroinflammatory signaling upregulation in Alzheimer's disease. Neurochem. Res. 25, 1173-1184.

Lund, E. G., Guileyardo, J. M., and Russell, D. W. (1999). cDNA cloning of cholesterol 24-hydroxylase, a mediator of cholesterol homeostasis in the brain. Proc. Natl. Acad. Sci. U.S.A. 96, 7238-7243. doi: 10.1073/pnas.96.13.7238

Lyons, M. A., and Brown, A. J. (2000). Metabolism of 7-ketocholesterol by sterol 27-hydroxylase in human hepatic cells. Atherosclerosis 151:291. doi: 10.1016/ s0021-9150(00)81320-9

Ma, D. W., Ngo, V., Huot, P. S., and Kang, J. X. (2006). N-3 polyunsaturated fatty acids endogenously synthesized in fat-1 mice are enriched in the mammary gland. Lipids 41, 35-39. doi: 10.1007/s11745-006-5067-9

Mahley, R. W. (2016). Central nervous system lipoproteins: ApoE and regulation of cholesterol metabolism. Arterioscler. Thromb. Vasc. Biol. 36, 1305-1315. doi: 10.1161/ATVBAHA.116.307023

Mahley, R. W., and Rall, S. C. Jr. (2000). Apolipoprotein E: far more than a lipid transport protein. Annu. Rev. Genomics Hum. Genet. 1, 507-537. doi: 10.1146/annurev.genom.1.1.507

Malandrino, M. I., Fucho, R., Weber, M., Calderon-Dominguez, M., Mir, J. F., Valcarcel, L., et al. (2015). Enhanced fatty acid oxidation in adipocytes and macrophages reduces lipid-induced triglyceride accumulation and inflammation. Am. J. Physiol. Endocrinol. Metab. 308, E756-E769. doi: 10.1152/ ajpendo.00362.2014

Marquer, C., Devauges, V., Cossec, J. C., Liot, G., Lecart, S., Saudou, F., et al. (2011). Local cholesterol increase triggers amyloid precursor protein-Bacel clustering in lipid rafts and rapid endocytosis. FASEB J. 25, 1295-1305. doi: 10.1096/fj.10168633

Mathews, E. S., and Appel, B. (2016). Cholesterol biosynthesis supports myelin gene expression and axon ensheathment through modulation of P13K/Akt/mTor signaling. J. Neurosci. Off. J. Soc. Neurosci. 36, 7628-7639. doi: 10.1523/JNEUROSCI.0726-16.2016

Mathys, H., Adaikkan, C., Gao, F., Young, J. Z., Manet, E., Hemberg, M., et al. (2017). Temporal tracking of microglia activation in neurodegeneration at single-cell resolution. Cell Rep. 21, 366-380. doi: 10.1016/j.celrep.2017.09.039

Mauch, D. H., Nagler, K., Schumacher, S., Goritz, C., Muller, E. C., Otto, A., et al. (2001). CNS synaptogenesis promoted by glia-derived cholesterol. Science 294, 1354-1357. doi: 10.1126/science.294.5545.1354

Mauerer, R., Walczak, Y., and Langmann, T. (2009). Comprehensive mRNA profiling of lipid-related genes in microglia and macrophages using taqman arrays. Methods Mol. Biol. 580, 187-201. doi: 10.1007/978-1-60761-325-1_10

Mehta, M. M., Weinberg, S. E., and Chandel, N. S. (2017). Mitochondrial control of immunity: beyond ATP. Nat. Rev. Immunol. 17, 608-620. doi: 10.1038/nri. 2017.66

Mitiæ, T., Andrew, R., Walker, B. R., and Hadoke, P. W. F. (2013). 11ßHydroxysteroid dehydrogenase type 1 contributes to the regulation of 7 oxysterol levels in the arterial wall through the inter-conversion of 7 ketocholesterol and 7 $\beta$-hydroxycholesterol. Biochimie 95, 548-555. doi: 10 . 1016/j.biochi.2012.08.007

Mizutani, M., Pino, P. A., Saederup, N., Charo, I. F., Ransohoff, R. M., and Cardona, A. E. (2012). The fractalkine receptor but not CCR2 is present on microglia from embryonic development throughout adulthood. J. Immunol. 188, 29-36. doi: 10.4049/jimmunol.1100421

Morari, J., Anhe, G. F., Nascimento, L. F., de Moura, R. F., Razolli, D., Solon, C., et al. (2014). Fractalkine (CX3CL1) is involved in the early activation of hypothalamic inflammation in experimental obesity. Diabetes 63, 3770-3784. doi: $10.2337 / \mathrm{db} 13-1495$

Morell, Q. R. (1999). "Characteristic composition of myelin," in Basic Neurochemistry: Molecular, Cellular and Medical Aspects, eds A.B. Siegel and R. W. Albers (Philadelphia: Lippincott-Raven).

Mudo, G., Frinchi, M., Nuzzo, D., Scaduto, P., Plescia, F., Massenti, M. F., et al. (2019). Anti-inflammatory and cognitive effects of interferon-betala (IFNbeta1a) in a rat model of Alzheimer's disease. J. Neuroinflammation 16:44. doi: 10.1186/s12974-019-1417-4

Murphy, E. J. (2017). The blood-brain barrier and protein-mediated fatty acid uptake: role of the blood-brain barrier as a metabolic barrier: an editorial comment for 'The blood-brain barrier fatty acid transport protein 1 (FATP1/SLC27A1) supplies docosahexaenoic acid to the brain, and insulin facilitates transport'. J. Neurochem. 141, 324-329. doi: 10.1111/jnc. 14000

Namgaladze, D., and Brune, B. (2016). Macrophage fatty acid oxidation and its roles in macrophage polarization and fatty acid-induced inflammation. Biochim. Biophys. Acta 1861, 1796-1807. doi: 10.1016/j.bbalip.2016.09.002

Namgaladze, D., Lips, S., Leiker, T. J., Murphy, R. C., Ekroos, K., Ferreiros, N., et al. (2014). Inhibition of macrophage fatty acid beta-oxidation exacerbates palmitate-induced inflammatory and endoplasmic reticulum stress responses. Diabetologia 57, 1067-1077. doi: 10.1007/s00125-014-3173-4

Naruse, M., Ishizaki, Y., Ikenaka, K., Tanaka, A., and Hitoshi, S. (2017). Origin of oligodendrocytes in mammalian forebrains: a revised perspective. J. Physiol. Sci. JPS 67, 63-70. doi: 10.1007/s12576-016-0479-7

Nathanael, J. S., Garmire, L. X., McDonald, J. G., Myers, D. S., Milne, S. B., Shibata, N., et al. (2012). Regulated accumulation of desmosterol integrates macrophage lipid metabolism and inflammatory responses. Cell 151, 138-152. doi: 10.1016/j.cell.2012.06.054

Nau, R., Ribes, S., Djukic, M., and Eiffert, H. (2014). Strategies to increase the activity of microglia as efficient protectors of the brain against infections. Front. Cell. Neurosci. 8:138. doi: 10.3389/fncel.2014.00138

Nguyen, L. N., Ma, D., Shui, G., Wong, P., Cazenave-Gassiot, A., Zhang, X., et al. (2014). Mfsd2a is a transporter for the essential omega-3 fatty acid docosahexaenoic acid. Nature 509, 503-506. doi: 10.1038/nature 13241

Nieweg, K., Schaller, H., and Pfrieger, F. W. (2009). Marked differences in cholesterol synthesis between neurons and glial cells from postnatal rats. J. Neurochem. 109, 125-134. doi: 10.1111/j.1471-4159.2009.05917.x

Nimmerjahn, A., Kirchhoff, F., and Helmchen, F. (2005). Resting microglial cells are highly dynamic surveillants of brain parenchyma in vivo. Science 308, 1314-1318. doi: 10.1126/science.1110647

Nishitsuji, K., Hosono, T., Uchimura, K., and Michikawa, M. (2011). Lipoprotein lipase is a novel amyloid beta (Abeta)-binding protein that promotes glycosaminoglycan-dependent cellular uptake of Abeta in astrocytes. J. Biol. Chem. 286, 6393-6401. doi: 10.1074/jbc.M110.172106

Ochocka, N., Segit, P., Walentynowicz, K. A., Wojnicki, K., Cyranowski, S., Swatler, J., et al. (2019). Single-cell RNA sequencing reveals functional heterogeneity and sex differences of glioma-associated brain macrophages. bioRxiv [Preprint]. doi: 10.1101/752949

Olah, M., Amor, S., Brouwer, N., Vinet, J., Eggen, B., Biber, K., et al. (2012). Identification of a microglia phenotype supportive of remyelination. Glia 60, 306-321. doi: 10.1002/glia.21266

Pan, X.-D., Zhu, Y.-G., Lin, N., Zhang, J., Ye, Q.-Y., Huang, H.-P., et al. (2011). Microglial phagocytosis induced by fibrillar $\beta$-amyloid is attenuated by oligomeric $\beta$-amyloid: implications for Alzheimer's disease. Mol. Neurodegener. 6:45. doi: 10.1186/1750-1326-6-45

Panayiotou, E., Fella, E., Papacharalambous, R., Malas, S., Saraiva, M. J., and Kyriakides, T. (2017). C1q ablation exacerbates amyloid deposition: a study in a transgenic mouse model of ATTRV30M amyloid neuropathy. PLoS One 12:e0175767. doi: 10.1371/journal.pone.0175767

Paolicelli, R. C., Bolasco, G., Pagani, F., Maggi, L., Scianni, M., Panzanelli, P., et al. (2011). Synaptic pruning by microglia is necessary for normal brain development. Science 333:1456. doi: 10.1126/science.1202529 
Pocivavsek, A., Burns, M. P., and Rebeck, G. W. (2009). Low-density lipoprotein receptors regulate microglial inflammation through c-Jun N-terminal kinase. Glia 57, 444-453. doi: 10.1002/glia. 20772

Posokhova, E. N., Khoshchenko, O. M., Chasovskikh, M. I., Pivovarova, E. N., and Dushkin, M. I. (2008). Lipid synthesis in macrophages during inflammation in vivo: effect of agonists of peroxisome proliferator activated receptors alpha and gamma and of retinoid X receptors. Biochemistry (Mosc.) 73, 296-304. doi: 10.1134/s0006297908030097

Prinz, M., Erny, D., and Hagemeyer, N. (2017). Ontogeny and homeostasis of CNS myeloid cells. Nat. Immunol. 18:385. doi: 10.1038/ni.3703

Purdon, A. D., Rosenberger, T. A., Shetty, H. U., and Rapoport, S. I. (2002). Energy consumption by phospholipid metabolism in mammalian brain. Neurochem. Res. 27, 1641-1647.

Raine, C. S., Scheinberg, L., and Waltz, J. M. (1981). Multiple sclerosis. Oligodendrocyte survival and proliferation in an active established lesion. Lab. Invest. 45, 534-546.

Rambold, A. S., and Pearce, E. L. (2018). Mitochondrial dynamics at the interface of immune cell metabolism and function. Trends Immunol. 39, 6-18. doi: 10. 1016/j.it.2017.08.006

Ransohoff, R. M. (2016). A polarizing question: do M1 and M2 microglia exist? Nat. Neurosci. 19, 987-991. doi: 10.1038/nn.4338

Rapoport, S. I. (1999). In vivo fatty acid incorporation into brain phospholipids in relation to signal transduction and membrane remodeling. Neurochem. Res. 24, $1403-1415$.

Reale, M., and Sanchez-Ramon, S. (2017). Lipids at the cross-road of autoimmunity in multiple sclerosis. Curr. Med. Chem. 24, 176-192. doi: 10. 2174/0929867324666161123093606

Reichwald, J., Danner, S., Wiederhold, K. H., and Staufenbiel, M. (2009). Expression of complement system components during aging and amyloid deposition in APP transgenic mice. J. Neuroinflammation 6:35. doi: 10.1186/ 1742-2094-6-35

Ren, L., and Ren, X. (2016). Meta-analyses of four polymorphisms of lipoprotein lipase associated with the risk of Alzheimer's disease. Neurosci. Lett. 619, 73-78. doi: 10.1016/j.neulet.2016.03.021

Riddell, D. R., Christie, G., Hussain, I., and Dingwall, C. (2001).Compartmentalization of beta-secretase (Asp2) into lowbuoyant density, noncaveolar lipid rafts. Curr. Biol. 11, 1288-1293. doi: 10.1016/s0960-9822(01)00394-3

Robinson, P. J., Noronha, J., DeGeorge, J. J., Freed, L. M., Nariai, T., and Rapoport, S. I. (1992). A quantitative method for measuring regional in vivo fatty-acid incorporation into and turnover within brain phospholipids: review and critical analysis. Brain Res. Brain Res. Rev. 17, 187-214. doi: 10.1016/0165-0173(92) 90016- $\mathrm{f}$

Rosenbauer, F., and Tenen, D. G. (2007). Transcription factors in myeloid development: balancing differentiation with transformation. Nat. Rev. Immunol. 7, 105-117. doi: 10.1038/nri2024

Ross, B. M., Mamalias, N., Moszczynska, A., Rajput, A. H., and Kish, S. J. (2001). Elevated activity of phospholipid biosynthetic enzymes in substantia nigra of patients with Parkinson's disease. Neuroscience 102, 899-904. doi: 10.1016/ s0306-4522(00)00501-7

Saher, G., Brugger, B., Lappe-Siefke, C., Mobius, W., Tozawa, R., Wehr, M. C., et al. (2005). High cholesterol level is essential for myelin membrane growth. Nat. Neurosci. 8, 468-475. doi: 10.1038/nn1426

Salminen, A., and Kaarniranta, K. (2009). Siglec receptors and hiding plaques in Alzheimer's disease. J. Mol. Med. (Berl.) 87, 697-701. doi: 10.1007/s00109-0090472-1

Schafer, D. P., Lehrman, E. K., Kautzman, A. G., Koyama, R., Mardinly, A. R., Yamasaki, R., et al. (2012). Microglia sculpt postnatal neural circuits in an activity and complement-dependent manner. Neuron 74, 691-705. doi: 10. 1016/j.neuron.2012.03.026

Schwinkendorf, D. R., Tsatsos, N. G., Gosnell, B. A., and Mashek, D. G. (2011). Effects of central administration of distinct fatty acids on hypothalamic neuropeptide expression and energy metabolism. Int. J. Obes. (Lond.) 35, 336-344. doi: 10.1038/ijo.2010.159

Sekar, A., Bialas, A. R., de Rivera, H., Davis, A., Hammond, T. R., Kamitaki, N., et al. (2016). Schizophrenia risk from complex variation of complement component 4. Nature 530, 177-183. doi: 10.1038/nature16549
Sellgren, C. M., Gracias, J., Watmuff, B., Biag, J. D., Thanos, J. M., Whittredge, P. B., et al. (2019). Increased synapse elimination by microglia in schizophrenia patient-derived models of synaptic pruning. Nat. Neurosci. 22, 374-385. doi: 10.1038/s41593-018-0334-7

Serhan, C. N. (2014). Pro-resolving lipid mediators are leads for resolution physiology. Nature 510, 92-101. doi: 10.1038/nature13479

Shi, Q., Chowdhury, S., Ma, R., Le, K. X., Hong, S., Caldarone, B. J., et al. (2017). Complement $\mathrm{C} 3$ deficiency protects against neurodegeneration in aged plaquerich APP/PS1 mice. Sci. Transl. Med. 9:eaaf6295. doi: 10.1126/scitranslmed. aaf6295

Shirotani, K., Hori, Y., Yoshizaki, R., Higuchi, E., Colonna, M., Saito, T. et al. (2019). Aminophospholipids are signal-transducing TREM2 ligands on apoptotic cells. Sci. Rep. 9:7508. doi: 10.1038/s41598-019-43535-6

Siegert, E., Paul, F., Rothe, M., and Weylandt, K. H. (2017). The effect of omega3 fatty acids on central nervous system remyelination in fat- 1 mice. $B M C$ Neurosci. 18:19. doi: 10.1186/s12868-016-0312-5

Simopoulos, A. P. (2008). The importance of the omega-6/omega-3 fatty acid ratio in cardiovascular disease and other chronic diseases. Exp. Biol. Med. (Maywood) 233, 674-688. doi: 10.3181/0711-MR-311

Simopoulos, A. P. (2011). Evolutionary aspects of diet: the omega-6/omega-3 ratio and the brain. Mol. Neurobiol. 44, 203-215. doi: 10.1007/s12035-010-8162-0

Simopoulos, A. P. (2016). An increase in the omega-6/omega-3 fatty acid ratio Increases the risk for obesity. Nutrients 8:128. doi: 10.3390/nu8030128

Singh, S., Mishra, A., Srivastava, N., Shukla, R., and Shukla, S. (2018). Acetyl-1-Carnitine via upegulating dopamine D1 receptor and attenuating microglial activation prevents neuronal loss and improves memory functions in Parkinsonian Rats. Mol. Neurobiol. 55, 583-602. doi: 10.1007/s12035-0160293-5

Song, G. J., Nam, Y., Jo, M., Jung, M., Koo, J. Y., Cho, W., et al. (2016). A novel small-molecule agonist of PPAR-gamma potentiates an anti-inflammatory M2 glial phenotype. Neuropharmacology 109, 159-169. doi: 10.1016/j.neuropharm. 2016.06.009

Stukas, S., Robert, J., Lee, M., Kulic, I., Carr, M., Tourigny, K., et al. (2014). Intravenously injected human apolipoprotein A-I rapidly enters the central nervous system via the choroid plexus. J. Am. Heart Assoc. 3:e001156. doi: 10.1161/JAHA.114.001156

Taha, A. Y., Chang, L., and Chen, M. (2016). Threshold changes in rat brain docosahexaenoic acid incorporation and concentration following graded reductions in dietary alpha-linolenic acid. Prostaglandins Leukot. Essent. Fatty Acids 105, 26-34. doi: 10.1016/j.plefa.2015.12.002

Tambuyzer, B. R., Ponsaerts, P., and Nouwen, E. J. (2009). Microglia: gatekeepers of central nervous system immunology. J. Leukoc. Biol. 85, 352-370. doi: 10.1189/ jlb.0608385

Thaler, J. P., Yi, C. X., Schur, E. A., Guyenet, S. J., Hwang, B. H., Dietrich, M. O., et al. (2012). Obesity is associated with hypothalamic injury in rodents and humans. J. Clin. Invest. 122, 153-162. doi: 10.1172/JCI59660

Trépanier, M.-O., Hopperton, K. E., Orr, S. K., and Bazinet, R. P. (2016). N3 polyunsaturated fatty acids in animal models with neuroinflammation: an update. Eur. J. Pharmacol. 785, 187-206. doi: 10.1016/j.ejphar.2015.05.045

Tu, T. H., Kim, H., Yang, S., Kim, J. K., and Kim, J. G. (2019). Linoleic acid rescues microglia inflammation triggered by saturated fatty acid. Biochem. Biophys. Res. Commun. 513, 201-206. doi: 10.1016/j.bbrc.2019.03.047

Tu, Y. F., Lu, P. J., Huang, C. C., Ho, C. J., and Chou, Y. P. (2012). Moderate dietary restriction reduces p53-mediated neurovascular damage and microglia activation after hypoxic ischemia in neonatal brain. Stroke 43, 491-498. doi: 10.1161/STROKEAHA.111.629931

van de Kraats, C., Killestein, J., Popescu, V., Rijkers, E., Vrenken, H., Lütjohann, D., et al. (2013). Oxysterols and cholesterol precursors correlate to magnetic resonance imaging measures of neurodegeneration in multiple sclerosis. Mult. Scler. J. 20, 412-417. doi: 10.1177/1352458513499421

Van den Bossche, J., and Saraber, D. L. (2018). Metabolic regulation of macrophages in tissues. Cell. Immunol. 330, 54-59. doi: 10.1016/j.cellimm.2018.01.009

van der Kant, R., Langness, V. F., Herrera, C. M., Williams, D. A., Fong, L. K., Leestemaker, Y., et al. (2019). Cholesterol metabolism is a druggable axis that independently regulates tau and amyloid-beta in iPSC-derived Alzheimer's disease neurons. Cell Stem Cell 24:e9. doi: 10.1016/j.stem.2018.12.013

Van Hove, H., Martens, L., Scheyltjens, I., De Vlaminck, K., Pombo Antunes, A. R., Prijck, S. De, et al. (2019). A single-cell atlas of mouse brain macrophages 
reveals unique transcriptional identities shaped by ontogeny and tissue environment. Nat. Neurosci. 22, 1021-1035. doi: 10.1038/s41593-019-0393-4

Vance, J. E., Campenot, R. B., and Vance, D. E. (2000). The synthesis and transport of lipids for axonal growth and nerve regeneration. Biochim. Biophys. Acta 1486, 84-96. doi: 10.1016/s1388-1981(00)00050-0

Viader, A., Ogasawara, D., Joslyn, C. M., Sanchez-Alavez, M., Mori, S., Nguyen, W., et al. (2016). A chemical proteomic atlas of brain serine hydrolases identifies cell type-specific pathways regulating neuroinflammation. eLife 5:e12345. doi: 10.7554/eLife. 12345

Wake, H., Moorhouse, A. J., Jinno, S., Kohsaka, S., and Nabekura, J. (2009). Resting microglia directly monitor the functional state of synapses in vivo and determine the fate of ischemic terminals. J. Neurosci. 29, 3974-3980. doi: 10.1523/JNEUROSCI.4363-08.2009

Wang, M. D., Kiss, R. S., Franklin, V., McBride, H. M., Whitman, S. C., and Marcel, Y. L. (2007). Different cellular traffic of LDL-cholesterol and acetylated LDLcholesterol leads to distinct reverse cholesterol transport pathways. J. Lipid Res. 48, 633-645. doi: 10.1194/jlr.m600470-jlr200

Wang, Y., Cella, M., Mallinson, K., Ulrich, J. D., Young, K. L., Robinette, M. L., et al. (2015). TREM2 lipid sensing sustains the microglial response in an Alzheimer's disease model. Cell 160, 1061-1071. doi: 10.1016/j.cell.2015.01.049

Warburg, O., Wind, F., and Negelein, E. (1927). The metabolism of tumors in the body. J. Gen. Physiol. 8, 519-530. doi: 10.1085/jgp.8.6.519

Warshaw, J. B., and Terry, M. L. (1976). Cellular energy metabolism during fetal development. VI. Fatty acid oxidation by developing brain. Dev. Biol. 52, 161-166. doi: 10.1016/0012-1606(76)90016-6

Wei, L., Sun, C., Lei, M., Li, G., Yi, L., Luo, F., et al. (2013). Activation of Wnt/B-catenin pathway by exogenous Wnt1 protects SH-SY5Y cells against 6hydroxydopamine toxicity. J. Mol. Neurosci. 49, 105-115. doi: 10.1007/s12031012-9900-8

Weinhard, L., di Bartolomei, G., Bolasco, G., Machado, P., Schieber, N. L., Neniskyte, U., et al. (2018). Microglia remodel synapses by presynaptic trogocytosis and spine head filopodia induction. Nat. Commun. 9:1228. doi: 10.1038/s41467-018-03566-5

West, A. P., Brodsky, I. E., Rahner, C., Woo, D. K., Erdjument-Bromage, H., Tempst, P., et al. (2011). TLR signalling augments macrophage bactericidal activity through mitochondrial ROS. Nature 472, 476-480. doi: 10.1038/ nature 09973

Wisniewski, T., Castano, E. M., Golabek, A., Vogel, T., and Frangione, B. (1994). Acceleration of Alzheimer's fibril formation by apolipoprotein $\mathrm{E}$ in vitro. Am. J. Pathol. 145, 1030-1035.

Wlodarczyk, A., Holtman, I. R., Krueger, M., Yogev, N., Bruttger, J., Khorooshi, R., et al. (2017). A novel microglial subset plays a key role in myelinogenesis in developing brain. EMBO J. 36, 3292-3308. doi: 10.15252/embj.2016 96056

Woller, S. A., Choi, S.-H., An, E. J., Low, H., Schneider, D. A., Ramachandran, R., et al. (2018). Inhibition of neuroinflammation by AIBP: spinal effects upon facilitated pain states. Cell Rep. 23, 2667-2677. doi: 10.1016/j.celrep.2018. 04.110

Wong, B. H., Chan, J. P., Cazenave-Gassiot, A., Poh, R. W., Foo, J. C., Galam, D. L. A., et al. (2016). Mfsd2a is a transporter for the essential $\omega-3$ fatty acid docosahexaenoic acid (DHA) in eye and is important for photoreceptor cell development. J. Biol. Chem. 291, 10501-10514. doi: 10.1074/jbc.M116.721340

Wu, D., Sanin, D. E., Everts, B., Chen, Q., Qiu, J., Buck, M. D., et al. (2016). Type 1 interferons induce changes in core metabolism that are critical for immune function. Immunity 44, 1325-1336. doi: 10.1016/j.immuni.2016.06.006

Wu, L., and Zhao, L. (2016). ApoE2 and Alzheimer's disease: time to take a closer look. Neural. Regen. Res. 11, 412-413.
Xicoy, H., Wieringa, B., and Martens, G. J. M. (2019). The role of lipids in Parkinson's disease. Cells 8:27.

Xu, Q., Bernardo, A., Walker, D., Kanegawa, T., Mahley, R. W., and Huang, Y. (2006). Profile and regulation of apolipoprotein E (ApoE) expression in the CNS in mice with targeting of green fluorescent protein gene to the ApoE locus. J. Neurosci. 26, 4985-4994. doi: 10.1523/jneurosci.5476-05.2006

Yamashita, A., Hayashi, Y., Matsumoto, N., Nemoto-Sasaki, Y., Oka, S., Tanikawa, T., et al. (2014). Glycerophosphate/acylglycerophosphate acyltransferases. Biology 3, 801-830. doi: 10.3390/biology3040801

Yang, D. S., Stavrides, P., Saito, M., Kumar, A., Rodriguez-Navarro, J. A., Pawlik, M., et al. (2014). Defective macroautophagic turnover of brain lipids in the TgCRND8 Alzheimer mouse model: prevention by correcting lysosomal proteolytic deficits. Brain 137, 3300-3318. doi: 10.1093/brain/awu278

Ye, J., and DeBose-Boyd, R. A. (2011). Regulation of cholesterol and fatty acid synthesis. Cold Spring Harb. Perspect. Biol. 3:a004754. doi: 10.1101/cshperspect. a004754

Yeh, F. L., Wang, Y., Tom, I., Gonzalez, L. C., and Sheng, M. (2016). TREM2 binds to apolipoproteins, including APOE and CLU/APOJ, and thereby facilitates uptake of amyloid-beta by microglia. Neuron 91, 328-340. doi: 10.1016/j. neuron.2016.06.015

Zhang, E., Kim, J.-J., Shin, N., Yin, Y., Nan, Y., Xu, Y., et al. (2017). High omega3 polyunsaturated fatty acids in fat-1 mice reduce inflammatory pain. J. Med. Food 20, 535-541. doi: 10.1089/jmf.2016.3871

Zhang, J., Zhang, X., Wang, L., and Yang, C. (2017). High performance liquid chromatography-mass spectrometry (LC-MS) based quantitative lipidomics study of ganglioside-NANA-3 plasma to establish its association with Parkinson's disease patients. Med. Sci. Monit. 23, 5345-5353. doi: 10.12659/ msm.904399

Zhang, Y., Chen, K., Sloan, S. A., Bennett, M. L., Scholze, A. R., O’Keeffe, S., et al. (2014). An RNA-sequencing transcriptome and splicing database of glia, neurons, and vascular cells of the cerebral cortex. J. Neurosci. 34, 11929-11947. doi: 10.1523/JNEUROSCI.1860-14.2014

Zhang, Y., Sloan, S. A., Clarke, L. E., Caneda, C., Plaza, C. A., Blumenthal, P. D., et al. (2016). Purification and characterization of progenitor and mature human astrocytes reveals transcriptional and functional differences with mouse. Neuron 89, 37-53. doi: 10.1016/j.neuron.2015.11.013

Zhao, C., and Dahlman-Wright, K. (2010). Liver X receptor in cholesterol metabolism. J. Endocrinol. 204, 233-240. doi: 10.1677/JOE-09-0271

Ziermans, T. B., Schothorst, P. F., Schnack, H. G., Koolschijn, P. C., Kahn, R. S., van Engeland, H., et al. (2012). Progressive structural brain changes during development of psychosis. Schizophr. Bull. 38, 519-530. doi: 10.1093/schbul/ sbq113

Ziko, I., De Luca, S., Dinan, T., Barwood, J. M., Sominsky, L., Cai, G., et al. (2014). Neonatal overfeeding alters hypothalamic microglial profiles and central responses to immune challenge long-term. Brain. Behav. Immun. 41, 32-43. doi: 10.1016/j.bbi.2014.06.014

Conflict of Interest: The authors declare that the research was conducted in the absence of any commercial or financial relationships that could be construed as a potential conflict of interest.

Copyright (c) 2020 Loving and Bruce. This is an open-access article distributed under the terms of the Creative Commons Attribution License (CC BY). The use, distribution or reproduction in other forums is permitted, provided the original author(s) and the copyright owner(s) are credited and that the original publication in this journal is cited, in accordance with accepted academic practice. No use, distribution or reproduction is permitted which does not comply with these terms. 